\title{
Associated production of heavy quarkonia and electroweak bosons at present and future colliders
}

\author{
Bernd A. Kniehl* \\ CERN, Theoretical Physics Division, 1211 Geneva 23, Switzerland \\ Caesar P. Palisoc \\ National Institute of Physics, University of the Philippines, Diliman, Quezon City 1101, Philippines \\ Lennart Zwirner \\ Institut für Theoretische Physik, Universität Hamburg, Luruper Chaussee 149, 22761 Hamburg, Germany
}

(Received 11 August 2002; published 17 December 2002)

\begin{abstract}
We investigate the associated production of heavy quarkonia, with angular-momentum quantum numbers ${ }^{2 S+1} L_{J}={ }^{1} S_{0},{ }^{3} S_{1},{ }^{1} P_{1},{ }^{3} P_{J}(J=0,1,2)$, and photons, $Z$ bosons, and $W$ bosons in photon-photon, photonhadron, and hadron-hadron collisions within the factorization formalism of nonrelativistic quantum chromodynamics providing all contributing partonic cross sections in analytic form. In the case of photoproduction, we also include the resolved-photon contributions. We present numerical results for the processes involving $J / \psi$ and $\chi_{c J}$ mesons appropriate for the Fermilab Tevatron, CERN LHC, DESY TESLA, operated in the $e^{+} e^{-}$ and $\gamma \gamma$ modes, and DESY THERA.
\end{abstract}

DOI: 10.1103/PhysRevD.66.114002

PACS number(s): 14.40.Gx, 12.38.Bx, 13.60.Le, 13.85.Ni

\section{INTRODUCTION}

Since its discovery in 1974 , the $J / \psi$ meson has provided a useful laboratory for quantitative tests of quantum chromodynamics (QCD) and, in particular, of the interplay of perturbative and nonperturbative phenomena. The factorization formalism of nonrelativistic QCD (NRQCD) [1] provides a rigorous theoretical framework for the description of heavyquarkonium production and decay. This formalism implies a separation of short-distance coefficients, which can be calculated perturbatively as expansions in the strong-coupling constant $\alpha_{s}$, from long-distance matrix elements (MEs), which must be extracted from experiment. The relative importance of the latter can be estimated by means of velocity scaling rules; i.e., the MEs are predicted to scale with a definite power of the heavy-quark $(Q)$ velocity $v$ in the limit $v$ $\ll 1$. In this way, the theoretical predictions are organized as double expansions in $\alpha_{s}$ and $v$. A crucial feature of this formalism is that it takes into account the complete structure of the $Q \bar{Q}$ Fock space, which is spanned by the states $n$ $={ }^{2 S+1} L_{J}^{(c)}$ with definite spin $S$, orbital angular momentum $L$, total angular momentum $J$, and color multiplicity $c$ $=1,8$. In particular, this formalism predicts the existence of color-octet $(\mathrm{CO})$ processes in nature. This means that $Q \bar{Q}$ pairs are produced at short distances in $\mathrm{CO}$ states and subsequently evolve into physical, color-singlet (CS) quarkonia by the nonperturbative emission of soft gluons. In the limit $v$ $\rightarrow 0$, the traditional CS model (CSM) [2] is recovered. The greatest triumph of this formalism was that it was able to correctly describe [3] the cross section of inclusive charmonium hadroproduction measured in $p \bar{p}$ collisions at the Fermilab Tevatron [4], which had turned out to be more than

\footnotetext{
*Permanent address: II. Institut für Theoretische Physik, Universität Hamburg, Luruper Chaussee 149, 22761 Hamburg, Germany.
}

one order of magnitude in excess of the theoretical prediction based on the CSM.

In order to convincingly establish the phenomenological significance of the $\mathrm{CO}$ processes, it is indispensable to identify them in other kinds of high-energy experiments as well. Studies of charmonium production in ep photoproduction, e $p$ and $\nu N$ deep-inelastic scattering, $e^{+} e^{-}$annihilation, $\gamma \gamma$ collisions, and $b$-hadron decays may be found in the literature; see Ref. [5] and references cited therein. Furthermore, the polarization of charmonium, which also provides a sensitive probe of $\mathrm{CO}$ processes, was investigated [6,7]. Until very recently, none of these studies were able to prove or disprove the NRQCD factorization hypothesis [1]. However, preliminary data of $\gamma \gamma \rightarrow J / \psi+X$ taken by the DELPHI Collaboration [8] at LEP2 provide the first independent evidence for it [9].

In most NRQCD studies of charmonium production in high-energy particle collisions, the charmonium state is produced either singly or in association with a hadron jet $(j)$, which originates from an outgoing quark $(q)$ or gluon $(g)$, because this is bound to yield the largest cross sections $[2,3,6,7,9]$. However, also the associated production of $J / \psi$ mesons and prompt photons $(\gamma)$ was considered, in photonphoton [10-13], photon-hadron [14,15], and hadron-hadron collisions $[15,16]$. Although the cross sections of the latter processes are suppressed by a factor of $\alpha / \alpha_{s}$, where $\alpha$ is Sommerfeld's fine-structure constant, relative to the case of $J / \psi+j$ associated production, these processes exhibit some attractive features. On the one hand, $J / \psi+\gamma$ associated production in photon-photon collisions dominantly proceeds through the CS partonic subprocesses of direct and doubly resolved photoproduction, $\quad \gamma \gamma \rightarrow c \bar{c}\left[{ }^{3} S_{1}^{(1)}\right] \gamma$ and $g g$ $\rightarrow c \bar{c}\left[{ }^{3} S_{1}^{(1)}\right] \gamma$, respectively, and thus allows for an independent determination of the $\operatorname{CS} \operatorname{ME}\left\langle\mathcal{O}^{J / \psi}\left[{ }^{3} S_{1}^{(1)}\right]\right\rangle$, which is usually extracted from the measured leptonic annihilation rate of the $J / \psi$ meson. On the other hand, $J / \psi+\gamma$ associated 
production in photon-hadron collisions provides a good handle on the $\mathrm{CO}$ mechanism because the partonic subprocesses of direct photoproduction, $\gamma g \rightarrow c \bar{c}[n] \gamma$ with $n$ $={ }^{1} S_{0}^{(8)},{ }^{3} S_{1}^{(8)},{ }^{3} P_{J}^{(8)}$, are pure CO processes $[14,15]$. Finally, for small $J / \psi$ transverse momentum, $J / \psi+\gamma$ associated hadroproduction chiefly proceeds through the CS partonic subprocess $g g \rightarrow c \bar{c}\left[{ }^{3} S_{1}^{(1)}\right] \gamma$ and thus lends itself as a clean probe of the gluon density inside the proton $[15,16]$.

The purpose of this paper is to study the associated production of heavy quarkonia with the intermediate bosons, $W$ and $Z$, in photon-photon, photon-hadron, and hadron-hadron collisions to leading order (LO) in the NRQCD factorization formalism. We consider all experimentally established heavy quarkonia, which are classified by their angular-momentum quantum numbers ${ }^{2 S+1} L_{J}={ }^{1} S_{0},{ }^{3} S_{1},{ }^{1} P_{1},{ }^{3} P_{J}$ with $J$ $=0,1,2$. In the case of charmonium, these correspond to the $\eta_{c}, J / \psi\left(\psi^{\prime}, \psi(3 S)\right.$, etc. $), h_{c}$, and $\chi_{c J}$ mesons, respectively. We present all contributing partonic cross sections in analytic form. As a by-product of our analysis, we recover the corresponding formulas for the associated production of heavy quarkonia with prompt photons, by appropriately adjusting the couplings and mass of the $Z$ boson. In our numerical study, we concentrate on those charmonia which can be most straightforwardly identified experimentally, namely the $J / \psi$ and $\chi_{c J}$ mesons, through their leptonic and radiative decays, respectively. Specifically, we assess the feasibilities of the $p \bar{p}$ synchrotron Tevatron, which is in operation at Fermilab, the $p p$ synchrotron Large Hadron Collider (LHC), which is under construction at CERN, the TeV Energy Superconduction Linear Accelerator (TESLA), which is being developed and planned at DESY and can be operated in the $e^{+} e^{-}$[17] and $\gamma \gamma[18]$ modes, and the $p e^{ \pm}$collider THERA, which uses the TESLA lepton beam and the proton beam of the DESY ep collider HERA [19], to produce $J / \psi$ and $\chi_{c J}$ mesons in association with prompt photons, $W$ bosons, and $Z$ bosons. These colliders all operate with high luminosities at high energies and, at first sight, have the potential to produce these final states with reasonable rates. Furthermore, if one selects the leptonic decays of the $J / \psi$ meson and intermediate bosons, then these final states lead to spectacular signals, consisting of energetic isolated prompt photons, charged leptons, and possibly missing four-momentum, which should be easy to detect even at hadron colliders. A first step in this direction was recently undertaken in Ref. [20], which contains a numerical study of $Y+W$ and $Y+Z$ hadroproduction at the Tevatron and the LHC.

For completeness, we also provide numerical results for the cross sections of the production of $J / \psi$ and $\chi_{c J}$ mesons together with standard-model (SM) Higgs bosons, which are suppressed by the smallness of the charm Yukawa coupling. In fact, explicit analysis reveals that these cross sections are too small to produce any signals for the envisaged luminosities of the considered colliders, even in the case of the LHC. By the same token, the observation of events where a scalar boson is produced together with charmonium would signal new physics beyond the SM.

This paper is organized as follows. In Sec. II, we present our analytic results and explain how to evaluate the cross sections of the various processes enumerated above. The contributing partonic cross sections are collected in the Appendix. In Sec. III, we present our numerical results. Our conclusions are summarized in Sec. IV.

\section{ANALYTIC RESULTS}

In this section, we explain how to calculate the cross sections of the associated production of a heavy quarkonium state $C$ and an electroweak boson $D$ by photoproduction in $e^{+} e^{-}$and $p e^{ \pm}$collisions and by hadroproduction in $p \bar{p}$ and $p p$ collisions.

To start with, we consider $e^{+} e^{-}$collisions, where photons are unavoidably generated by hard initial-state bremsstrahlung. At a high-energy $e^{+} e^{-}$LC, an additional source of hard photons is provided by beamstrahlung, the synchrotron radiation emitted by one of the colliding bunches in the field of the opposite bunch. Bremsstrahlung and beamstrahlung both occur in the $e^{+} e^{-}$mode, and their energy spectra must be added coherently. The highest possible photon energies with large enough luminosity may be achieved by converting the $e^{+} e^{-}$LC into a $\gamma \gamma$ collider via back-scattering of highenergetic laser light off the electron and positron beams.

The photons can interact either directly with the quarks participating in the hard-scattering process (direct photoproduction) or via their quark and gluon content (resolved photoproduction). Thus, the inclusive process $e^{+} e^{-}$ $\rightarrow e^{+} e^{-} C D+X$, where $X$ denotes the hadronic remnant, receives contributions from the direct, singly resolved, and doubly resolved channels. All three contributions are formally of the same order in the perturbative expansion and must be included. This may be understood by observing that the parton density functions (PDFs) of the photon have a leading behavior proportional to $\alpha \ln \left(M^{2} / \Lambda_{\mathrm{QCD}}^{2}\right) \propto \alpha / \alpha_{s}$, where $M$ is the factorization scale and $\Lambda_{\mathrm{QCD}}$ is the asymptotic scale parameter of QCD.

Let us now describe how to calculate the cross section. We take the electron and positron to be massless and denote the masses of $C$ and $D$ by $m_{C}$ and $m_{D}$, respectively. Let $\sqrt{S}$ be the energy of the initial state, $y_{C}$ and $y_{D}$ the rapidities of $C$ and $D$, respectively, and $p_{T}$ their common transverse momentum in the center-of-mass (c.m.) frame of the collision. Invoking the Weizsäcker-Williams approximation (WWA) for electromagnetic bremsstrahlung [21], its analogues for beamstrahlung [23,24] and Compton back-scattering [25,26], and the factorization theorems of the QCD parton model [22] and NRQCD [1], the differential cross section of $e^{+} e^{-}$ $\rightarrow e^{+} e^{-} C D+X$ can be written as

$$
\begin{aligned}
\frac{d^{3} \sigma}{d p_{T}^{2} d y_{C} d y_{D}}\left(e^{+} e^{-} \rightarrow\right. & \left.e^{+} e^{-} C D+X\right) \\
= & \int_{\bar{x}_{+}}^{1} d x_{+} f_{\gamma / e}\left(x_{+}\right) \int_{\bar{x}_{-}}^{1} d x_{-} f_{\gamma / e}\left(x_{-}\right) \\
& \times \sum_{a, b, n} x_{a} f_{a / \gamma}\left(x_{a}, M\right) x_{b} f_{b / \gamma}\left(x_{b}, M\right) \\
& \times\left\langle\mathcal{O}^{C}[n]\right\rangle \frac{d \sigma}{d t}(a b \rightarrow Q \bar{Q}[n]+D),(1)
\end{aligned}
$$


where it is summed over the active partons $a, b=\gamma, g, q, \bar{q}$, $f_{\gamma / e}\left(x_{ \pm}\right)$are the photon flux functions, $f_{a / \gamma}\left(x_{a}, M\right)$ and $f_{b / \gamma}\left(x_{b}, M\right)$ are the PDFs of the photon, $\left\langle\mathcal{O}^{C}[n]\right\rangle$ are the MEs of $C,(d \sigma / d t)(a b \rightarrow Q \bar{Q}[n]+D)$ are the differential partonic cross sections, and $x_{ \pm}$and $x_{a, b}$ are the fractions of longitudinal momentum that the emitted particles receive from the emitting ones. With the definition $f_{\gamma / \gamma}\left(x_{\gamma}, M\right)$ $=\delta\left(1-x_{\gamma}\right)$, the direct, singly resolved, and doubly resolved channels are all accommodated. We have

$$
\begin{gathered}
\bar{x}_{ \pm}=\frac{m_{T}^{C} \exp \left( \pm y_{C}\right)+m_{T}^{D} \exp \left( \pm y_{D}\right)}{\sqrt{S}}, \\
x_{a, b}=\frac{m_{T}^{C} \exp \left( \pm y_{C}\right)+m_{T}^{D} \exp \left( \pm y_{D}\right)}{x_{ \pm} \sqrt{S}},
\end{gathered}
$$

where $m_{T}^{C}=\sqrt{m_{C}^{2}+p_{T}^{2}}$ is the transverse mass of $C$ and similarly for $D$.

The partonic Mandelstam variables $s=\left(p_{a}+p_{b}\right)^{2}, t$ $=\left(p_{a}-p_{C}\right)^{2}$, and $u=\left(p_{a}-p_{D}\right)^{2}$ can be expressed in terms of $p_{T}, y_{C}$, and $y_{D}$, as

$$
\begin{aligned}
& s=\left(m_{T}^{C}\right)^{2}+\left(m_{T}^{D}\right)^{2}+2 m_{T}^{C} m_{T}^{D} \cosh \left(y_{C}-y_{D}\right), \\
& t=-p_{T}^{2}-m_{T}^{C} m_{T}^{D} \exp \left(y_{D}-y_{C}\right), \\
& u=-p_{T}^{2}-m_{T}^{C} m_{T}^{D} \exp \left(y_{C}-y_{D}\right),
\end{aligned}
$$

respectively. Notice that $s+t+u=m_{C}^{2}+m_{D}^{2}$ and $s p_{T}^{2}=t u$ $-m_{C}^{2} m_{D}^{2}$. The kinematically allowed ranges of $S, p_{T}, y_{C}$, and $y_{D}$ are

$$
\begin{aligned}
& S \geqslant\left(m_{C}+m_{D}\right)^{2}, \\
& 0 \leqslant p_{T} \leqslant \frac{1}{2} \sqrt{\frac{\lambda\left(S, m_{C}^{2}, m_{D}^{2}\right)}{S},} \\
&\left|y_{C}\right| \leqslant \operatorname{arcosh} \frac{S+m_{C}^{2}-m_{D}^{2}}{2 \sqrt{S} m_{T}^{C}}, \\
&-\ln \frac{\sqrt{S}-m_{T}^{C} \exp \left(-y_{C}\right)}{m_{T}^{D}} \leqslant y_{D} \leqslant \ln \frac{\sqrt{S}-m_{T}^{C} \exp \left(y_{C}\right)}{m_{T}^{D}},
\end{aligned}
$$

where $\lambda(x, y, z)=x^{2}+y^{2}+z^{2}-2(x y+y z+z x)$ is Källén's function.

Sometimes it may be interesting to consider the cross section differential in the $C D$ invariant mass $m_{C D}=\sqrt{s}$ rather than in $p_{T}$. Using

$$
p_{T}^{2}=\frac{r \cosh \left(y_{C}-y_{D}\right)-m_{C D}^{2}}{2 \sinh ^{2}\left(y_{C}-y_{D}\right)}-\frac{m_{C}^{2}+m_{D}^{2}}{2},
$$

where $r=\sqrt{m_{C D}^{4}+\left(m_{C}^{2}-m_{D}^{2}\right)^{2} \sinh ^{2}\left(y_{C}-y_{D}\right)}$, we find

$$
\frac{d p_{T}^{2}}{d m_{C D}^{2}}=\frac{m_{C D}^{2} \cosh \left(y_{C}-y_{D}\right)-r}{2 r \sinh ^{2}\left(y_{C}-y_{D}\right)} .
$$

Equations (6) and (7) are regular for $y_{C}=y_{D}$ and read then

$$
\begin{gathered}
p_{T}^{2}=\frac{\lambda\left(S, m_{C}^{2}, m_{D}^{2}\right)}{4 S}, \\
\frac{d p_{T}^{2}}{d m_{C D}^{2}}=\frac{1}{4}\left[1-\left(\frac{m_{C}^{2}-m_{D}^{2}}{m_{C D}^{2}}\right)^{2}\right],
\end{gathered}
$$

respectively. Multiplying Eq. (1) with Eq. (7), we obtain $\left(d^{3} \sigma / d m_{C D}^{2} d y_{C} d y_{D}\right)\left(e^{+} e^{-} \rightarrow e^{+} e^{-} C D+X\right)$. The kinematically allowed ranges of $m_{C D}, y_{C}-y_{D}$, and $\left(y_{C}\right.$ $\left.+y_{D}\right) / 2$ are

$$
\begin{gathered}
m_{C}+m_{D} \leqslant m_{C D} \leqslant \sqrt{S} \\
\left|y_{C}-y_{D}\right| \leqslant \operatorname{arcosh} \frac{m_{C D}^{2}-m_{C}^{2}-m_{D}^{2}}{2 m_{C} m_{D}} \\
-\ln \frac{\sqrt{S}}{m_{T}^{C} \exp \left[-\left(y_{C}-y_{D}\right) / 2\right]+m_{T}^{D} \exp \left[\left(y_{C}-y_{D}\right) / 2\right]} \\
\leqslant \frac{y_{C}+y_{D}}{2} \\
\leqslant \ln \frac{\sqrt{S}}{m_{T}^{C} \exp \left[\left(y_{C}-y_{D}\right) / 2\right]+m_{T}^{D} \exp \left[-\left(y_{C}-y_{D}\right) / 2\right]} .
\end{gathered}
$$

We work in the fixed-flavor-number scheme, i.e., we have $n_{f}=3$ active quark flavors $q=u, d, s$ if $Q=c$ and $n_{f}=4$ active quark flavors $q=u, d, s, c$ if $Q=b$. As required by parton-model kinematics, we treat the $q$ quarks as massless. To LO in $v$, we need to include the $c \bar{c}$ Fock states $n={ }^{1} S_{0}^{(1)},{ }^{1} S_{0}^{(8)},{ }^{3} S_{1}^{(8)},{ }^{1} P_{1}^{(8)} \quad$ if $\quad H=\eta_{c} ; n$ $={ }^{3} S_{1}^{(1)},{ }^{1} S_{0}^{(8)},{ }^{3} S_{1}^{(8)},{ }^{3} P_{J}^{(8)}$ if $H=J / \psi, \psi^{\prime}, \psi(3 S), \ldots ; n$ $={ }^{1} P_{1}^{(1)},{ }^{1} S_{0}^{(8)}$ if $H=h_{c}$; and $n={ }^{3} P_{J}^{(1)},{ }^{3} S_{1}^{(8)}$ if $H=\chi_{c J}$, where $J=0,1,2[1]$. Their MEs satisfy the multiplicity relations

$$
\begin{aligned}
\left\langle\mathcal{O}^{\psi(n S)}\left[{ }^{3} P_{J}^{(8)}\right]\right\rangle & =(2 J+1)\left\langle\mathcal{O}^{\psi(n S)}\left[{ }^{3} P_{0}^{(8)}\right]\right\rangle, \\
\left\langle\mathcal{O}^{\chi_{c J}}\left[{ }^{3} P_{J}^{(1)}\right]\right\rangle & =(2 J+1)\left\langle\mathcal{O}^{\chi_{c 0}[}\left[{ }^{3} P_{0}^{(1)}\right]\right\rangle, \\
\left\langle\mathcal{O}^{\chi_{c J}}\left[{ }^{3} S_{1}^{(8)}\right]\right\rangle & =(2 J+1)\left\langle\mathcal{O}^{\chi_{c 0}[}\left[{ }^{3} S_{1}^{(8)}\right]\right\rangle,
\end{aligned}
$$

which follow to LO in $v$ from heavy-quark spin symmetry. The assignments for the various bottomonia are analogous.

The cross section of $p e^{ \pm} \rightarrow e^{ \pm} C D+X$ in photoproduction emerges from Eq. (1) by substituting $f_{\gamma / e}\left(x_{+}\right)=\delta\left(1-x_{+}\right)$ and replacing the photon PDFs $f_{a / \gamma}\left(x_{a}, M\right)$ with their proton counterparts $f_{a / p}\left(x_{a}, M\right)$. It receives contributions from the direct and resolved channels. Here, it is understood that 
$f_{\gamma / e}\left(x_{-}\right)$only accounts for electromagnetic bremsstrahlung. To conform with HERA conventions, we take the rapidities to be positive in the proton flight direction. The rapidity $y_{C}^{\text {lab }}$ of $C$ in the laboratory frame, where the proton and electron have energies $E_{p}$ and $E_{e}$, respectively, is related to $y_{C}$ by

$$
y_{C}^{\mathrm{lab}}=y_{C}+\frac{1}{2} \ln \frac{E_{p}}{E_{e}},
$$

and similarly for $D$. The c.m. energy is $\sqrt{S}=2 \sqrt{E_{p} E_{e}}$.

In a second step, the cross section of $p \bar{p} \rightarrow C D+X$ in hadroproduction is obtained from the one of $p e^{ \pm} \rightarrow e^{ \pm} C D$ $+X$ in photoproduction by substituting $f_{\gamma / e}\left(x_{-}\right)=\delta(1$ $\left.-x_{-}\right)$and replacing the photon PDFs $f_{b / \gamma}\left(x_{b}, M\right)$ with their antiproton counterparts $f_{b / \bar{p}}\left(x_{b}, M\right)$. In hadron-collider experiments, the $p \bar{p}$ c.m. frame and the laboratory one usually coincide. The cross section of $p p \rightarrow C D+X$ in hadroproduction is accordingly evaluated with $f_{b / p}\left(x_{b}, M\right)$.

We now turn to the partonic subprocesses $a b$ $\rightarrow Q \bar{Q}[n] D$. The differential cross section of such a process is calculated from the pertaining transition-matrix element $\mathcal{T}$ as $d \sigma / d t=\mid \overline{\left.\mathcal{T}\right|^{2}} /\left(16 \pi s^{2}\right)$, where the average is over the spin and color degrees of freedom of $a$ and $b$ and the spin of $D$ is summed over. We apply the covariant-projector method of Ref. [27] to implement the $Q \bar{Q}$ Fock states $n$ according to the NRQCD factorization formalism [1].

The following partonic subprocesses contribute to LO in $\alpha_{s}$ and $v$ :

$$
\begin{gathered}
\gamma \gamma \rightarrow Q \bar{Q}\left[s^{(1)}\right] N, \\
\gamma g \rightarrow Q \bar{Q}\left[s^{(8)}\right] N, \\
g g \rightarrow Q \bar{Q}\left[s^{(1)}\right] N, \\
g g \rightarrow Q \bar{Q}\left[s^{(8)}\right] N, \\
q \bar{q} \rightarrow Q \bar{Q}\left[s^{(8)}\right] N, \\
q_{u} \bar{q}_{d} \rightarrow Q \bar{Q}\left[{ }^{3} S_{1}^{(8)}\right] W^{+}, \\
q_{d} \bar{q}_{u} \rightarrow Q \bar{Q}\left[{ }^{3} S_{1}^{(8)}\right] W^{-},
\end{gathered}
$$

where $q=u, d, s, c ; q_{u}=u, c ; q_{d}=d, s ; N=\gamma, Z ;$ and $\mathrm{s}$ $={ }^{1} S_{0},{ }^{3} S_{1},{ }^{1} P_{1},{ }^{3} P_{J}$ with $J=0,1,2$. For the reason explained above, $q=c$ and $q_{u}=c$ must not be included if $Q$ $=c$. The processes $\gamma \gamma \rightarrow Q \bar{Q}\left[\varsigma^{(8)}\right] N$ and $\gamma g \rightarrow Q \bar{Q}\left[\varsigma^{(1)}\right] N$ are forbidden by color conservation. Furthermore, the processes $q \bar{q} \rightarrow Q \bar{Q}\left[\varsigma^{(1)}\right] N$ are prohibited because the $Q$-quark line is connected with the $q$-quark line by one gluon, which transmits color to the $Q \bar{Q}$ pair. For a similar reason and due to the fact that the $W$ boson must be emitted from the initialstate quarks if a $Q \bar{Q}$ pair is to be produced, processes (17) and (18) only come with $n={ }^{3} S_{1}^{(8)}$.

In the Higgs-boson case $D=H$, the contributing partonic subprocesses are analogous to Eqs. (12)-(16), except that, due to charge-conjugation invariance, $\gamma \gamma \rightarrow Q \bar{Q}\left[\varsigma^{(1)}\right] H$, $\gamma g \rightarrow Q \bar{Q}\left[\varsigma^{(8)}\right] H$, and $g g \rightarrow Q \bar{Q}\left[\varsigma^{(1)}\right] H$ are forbidden for $\varsigma$ $={ }^{3} S_{1},{ }^{1} P_{1}$, and $q \bar{q} \rightarrow Q \bar{Q}\left[\mathrm{~s}^{(8)}\right] H$ is forbidden for $\mathrm{s}$ $={ }^{1} S_{0},{ }^{3} P_{J}$.

The differential cross sections $d \sigma / d t$ of processes (12)(18) are listed in the Appendix. We combine the results proportional to the CO MEs $\left\langle\mathcal{O}^{\psi(n S)}\left[{ }^{3} P_{J}^{(8)}\right]\right\rangle$ and $\left\langle\mathcal{O}^{\chi_{c J}}\left[{ }^{3} S_{1}^{(8)}\right]\right\rangle$ exploiting the multiplicity relations of Eq. (9). We include large logarithmic corrections due to the running of the finestructure constant from the Thomson limit to the electroweak scale by expressing the gauge couplings in terms of Fermi's constant $G_{F}$, as $g=2^{1 / 4} G_{F}^{1 / 2} m_{Z}$ and $g^{\prime}=2^{3 / 4} G_{F}^{1 / 2} m_{W}$. Furthermore, we define the $Z q \bar{q}$ vector and axial-vector couplings as $v_{q}=I_{q}^{3}-2 e_{q} \sin ^{2} \theta_{w}$ and $a_{q}=I_{q}^{3}$, respectively, where $e_{q}$ is the fractional electric charge of quark $q, I_{q}^{3}$ is the third component of weak isospin of its left-handed component, and $\theta_{w}$ is the weak mixing angle, which is fixed by $\cos \theta_{w}$ $=m_{W} / m_{Z}$. The results for processes (13) and (14) may be obtained from those for process (12) by adjusting the overall color factors as specified in the Appendix. The results for $N=\gamma$ emerge from the ones for $N=Z$ by adjusting the couplings and mass of the $Z$ boson, by substituting $g=e, v_{q}$ $=e_{q}, a_{q}=0$, and $m_{Z}=0$, where $e=\sqrt{4 \pi \alpha}$ is the electron charge magnitude, and they are not presented separately. They vanish for processes (12)-(14) with $\varsigma={ }^{1} S_{0},{ }^{3} P_{J}$ and for process (16) with $\mathrm{s}={ }^{1} P_{1}$ because the photon has no axial-vector coupling.

In the case of $N=\gamma$, the differential cross sections $d \sigma / d t$ of processes (12)-(15) with $\varsigma={ }^{1} P_{1}$ and of process (16) with $\varsigma={ }^{3} S_{1},{ }^{3} P_{J}$ are plagued by infrared singularities in the limit of the final-state photon being soft, i.e., for $t=u=0$. In addition, process (16) with $\varsigma={ }^{3} S_{1}$ also suffers from a $u$ - or $t$-channel singularity if the final-state photon is hard and collinear to the initial-state $q$ or $\bar{q}$ quarks, i.e., for $t<u=0$ or $u<t=0$, respectively. Owing to the identity $p_{T}^{2}=t u / s$, both the soft and collinear limits entail $p_{T} \rightarrow 0$. By the same token, in the evaluations of Eq. (1) and its counterparts for lepton-hadron and hadron-hadron scattering, all these singularities can be avoided by imposing a lower cutoff on $p_{T}$. On the other hand, detailed inspection of these equations reveals that $d \sigma / d p_{T}$ is finite in the limit $p_{T} \rightarrow 0$ if $d \sigma / d t$ is [37]. The cases $D=Z, W, H$ are devoid of such singularities because $m_{D}$ acts as a regulator.

To our knowledge, the formulas presented in the Appendix cannot be found elsewhere in the literature. However, the literature contains analytic results for the partonic subprocesses pertinent to the case of $C=J / \psi$ and $D=\gamma$, namely, for processes (12) [10-12], (13) [14], and (14) [14,16] with $\varsigma={ }^{3} S_{1}$, and for processes (15) and (16) with $\varsigma$ ${ }^{1} S_{0},{ }^{3} S_{1},{ }^{3} P_{J}[14,16]$. We agree with them, except for Eq. (7) of Ref. [11] (see also related comments in Refs. $[9,12,13])$ and the term proportional to $\left\langle O_{8}^{J / \psi}\left({ }^{3} P_{0}\right)\right\rangle$ in Eq. (7) of Ref. [14] ([38]).

\section{NUMERICAL RESULTS}

We are now in a position to present our numerical results. We focus our attention on the cases $C=J / \psi, \chi_{c J}$. These 
TABLE I. Center-of-mass energies $\sqrt{S}$, design luminosities $L$, numbers of dedicated experiments, and total cross sections $\sigma_{1}$ corresponding to a yield of one signal event per year of operation for the considered colliders.

\begin{tabular}{lcccc}
\hline \hline Collider & $\sqrt{S}[\mathrm{TeV}]$ & $L\left[10^{32} \mathrm{~cm}^{-2} \mathrm{~s}^{-1}\right]$ & No. of experiments & $\sigma_{1}[\mathrm{fb}]$ \\
\hline TESLA $e^{+} e^{-}$mode & 0.5 & 340 & 1 & 0.0029 \\
TESLA $\gamma \gamma$ mode & 0.5 & 60 & 1 & 0.017 \\
THERA & 1 & 0.041 & 1 & 24 \\
Tevatron Run II & 2 & 2 & 2 & 0.25 \\
LHC & 14 & 100 & 2 & 0.005 \\
\hline \hline
\end{tabular}

charmonia can be efficiently identified experimentally, and their MEs are relatively well constrained [7]. The predicted cross-section distributions for the $\psi^{\prime}$ mesons are similar to those for the $J / \psi$ mesons, but their normalization is somewhat suppressed due to smaller MEs [7]. The $\eta_{c}$ meson is more difficult to detect experimentally, and the $h_{c}$ meson is poorly known [28].

We first describe our theoretical input and the kinematic conditions. We use $m_{c}=m_{C} / 2=1.5 \mathrm{GeV}$, $m_{W}=80.423 \mathrm{GeV}, \quad m_{Z}=91.1876 \mathrm{GeV}, \quad G_{F}=1.16639$ $\times 10^{-5} \mathrm{GeV}^{-2}, \quad \alpha=1 / 137.036$, and the LO formula for $\alpha_{s}^{\left(n_{f}\right)}(\mu)$ with $n_{f}=3$ active quark flavors [28]. We assume $m_{H}=115 \mathrm{GeV}$, a value just above the $95 \%$-confidence-level lower bound on $m_{H}$ from direct Higgs-boson searches and in agreement with tantalizing hints for the direct observation of the Higgs-boson signal delivered towards the end of the running phase of the CERN $e^{+} e^{-}$collider LEP2. As for the photon PDFs, we use the LO set from Glück, Reya, and Schienbein (GRS) [29], which is the only available one that is implemented in the fixed-flavor-number scheme, with $n_{f}$ $=3$. As for the proton PDFs, we use the LO set from Martin, Roberts, Stirling, and Thorne (MRST98LO) [30]. We adopt $\Lambda_{\mathrm{QCD}}^{(3)}=204 \mathrm{MeV}$ from Ref. [29], which happens to precisely correspond to $\Lambda_{\mathrm{QCD}}^{(4)}=174 \mathrm{MeV}$, the value employed in Ref. [30], if the matching scale is taken to be $m_{c}$. We choose the renormalization and factorization scales to be $\mu=M=m_{T}^{C}$ if $D=\gamma$ and to be $\mu=M=\sqrt{m_{T}^{C} m_{T}^{D}}$ if $D=Z, W, H$. As for the $J / \psi$ and $\chi_{c J}$ MEs, we adopt the set determined in Ref. [7] using the MRST98LO proton PDFs. Specifically, $\left.\left\langle\mathcal{O}^{J /} \psi^{3} S_{1}^{(1)}\right]\right\rangle$ and $\left\langle\mathcal{O}^{\chi_{c 0}[}\left[{ }^{3} P_{0}^{(1)}\right]\right\rangle$ were extracted from the measured partial decay widths of $J / \psi \rightarrow l^{+} l^{-}$and $\chi_{c 2} \rightarrow \gamma \gamma$ [28], respectively, while $\left\langle\mathcal{O}^{J /} \psi\left[{ }^{1} S_{0}^{(8)}\right]\right\rangle,\left\langle\mathcal{O}^{J / \psi}\left[{ }^{3} S_{1}^{(8)}\right]\right\rangle$, $\left.\left\langle\mathcal{O}^{J /} \psi^{3} P_{0}^{(8)}\right]\right\rangle$, and $\left\langle\mathcal{O}^{\chi_{c} 0}\left[{ }^{3} S_{1}^{(8)}\right]\right\rangle$ were fitted to the transverse-momentum distributions of $J / \psi$ and $\chi_{c J}$ inclusive hadroproduction [4] and the cross-section ratio $\sigma_{\chi_{c 2}} / \sigma_{\chi_{c 1}}$ [31] measured at the Tevatron. The fit results for $\left\langle\mathcal{O}^{J /} \psi\left[{ }^{1} S_{0}^{(8)}\right]\right\rangle$ and $\left\langle\mathcal{O}^{J / \psi}\left[{ }^{3} P_{0}^{(8)}\right]\right\rangle$ are strongly correlated, so that the linear combination

$$
M_{r}^{J / \psi}=\left\langle\mathcal{O}^{J / \psi}\left[{ }^{1} S_{0}^{(8)}\right]\right\rangle+\frac{r}{m_{c}^{2}}\left\langle\mathcal{O}^{J / \psi}\left[\left[{ }^{3} P_{0}^{(8)}\right]\right\rangle,\right.
$$

with a suitable value of $r$, is quoted. Unfortunately, Eq. (1) is sensitive to different linear combinations of $\left\langle\mathcal{O}^{J /} \psi^{1}\left[{ }^{1} S_{0}^{(8)}\right]\right\rangle$ and $\left\langle\mathcal{O}^{J / \psi}\left[{ }^{3} P_{0}^{(8)}\right]\right\rangle$ than appears in Eq. (19). In want of more specific information, we thus make the democratic choice $\left\langle\mathcal{O}^{J / \psi}\left[{ }^{1} S_{0}^{(8)}\right]\right\rangle=\left(r / m_{c}^{2}\right)\left\langle\mathcal{O}^{J / \psi}\left[{ }^{3} P_{0}^{(8)}\right]\right\rangle=M_{r}^{J / \psi} / 2$.

We now discuss the photon flux functions that enter our predictions for photoproduction at TESLA and THERA. The energy spectrum of the bremsstrahlung photons is well described in the WWA [21] by Eq. (27) of Ref. [32]. We assume that the scattered electrons and positrons will be antitagged, as was usually the case at LEP2, and take the maximum scattering angle to be $\theta_{\max }=25 \mathrm{mrad}$ [33]. The energy spectrum of the beamstrahlung photons is approximately described by Eq. (2.14) of Ref. [24]. It is controlled by the effective beamstrahlung parameter $Y$, which is given by Eq. (2.10) of that reference. Inserting the relevant TESLA parameters for the $\sqrt{S}=500 \mathrm{GeV}$ baseline design specified in Table 1.3.1 of Ref. [17] in that formula, we obtain $Y$ $=0.053$. In the case of the $e^{+} e^{-}$mode of TESLA, we coherently superimpose the WWA and beamstrahlung spectra, while, in the case of THERA, we only use the WWA spectrum. Finally, in the case of the $\gamma \gamma$ mode of TESLA, the energy spectrum of the back-scattered laser photons is given by Eq. (6a) of Ref. [25]. It depends on the parameter $\kappa$ $=s_{e \gamma} / m_{e}^{2}-1$, where $\sqrt{s_{e \gamma}}$ is the c.m. energy of the charged lepton and the laser photon, and it extends up to $x_{\max }=\kappa /(\kappa$ $+1)$, where $x$ is the energy of the back-scattered photons in units of $\sqrt{S} / 2$. The optimal value of $\kappa$ is $\kappa=2(1+\sqrt{2})$ $\approx 4.83$ [26], which we adopt; for larger values of $\kappa, e^{+} e^{-}$ pairs would be created in the collisions of laser and backscattered photons.

Since our study is at an exploratory level, we refrain from presenting a quantitative estimate of the theoretical uncertainties in our predictions. However, experience from previous analyses of charmonium production within the NRQCD factorization formalism $[9,34]$ leads us to expect relative errors of the order of $\pm 50 \%$.

Table I gives the c.m. energies $\sqrt{S}$, design luminosities $L$, numbers of dedicated experiments, and total cross sections $\sigma_{1}$ corresponding to a yield of one signal event per year of operation for the various colliders considered. At THERA, the proton and lepton energies in the laboratory frame are planned to be $E_{p}=1 \mathrm{TeV}$ and $E_{e}=250 \mathrm{GeV}$ [19]. One year of operation is usually taken to be $10^{7} \mathrm{~s}$, which corresponds to a duty factor of approximately $33 \%$ and can be achieved, e.g., by 200 days of running with an efficiency of $60 \%$. Thus, 


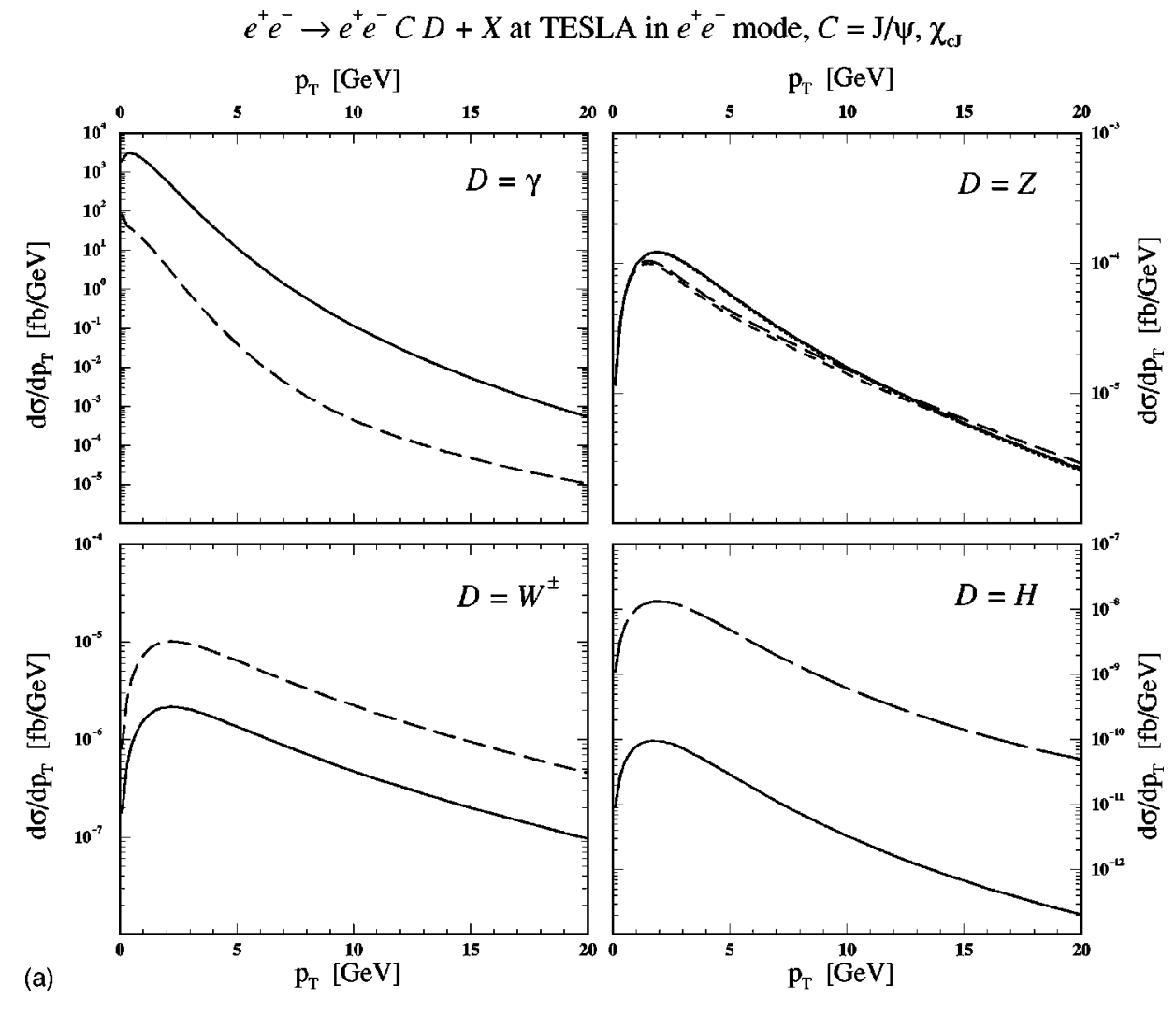

$e^{+} e^{-} \rightarrow e^{+} e^{-} C D+X$ at TESLA in $e^{+} e^{-}$mode, $C=\mathrm{J} / \psi, \chi_{\mathrm{cJ}}$

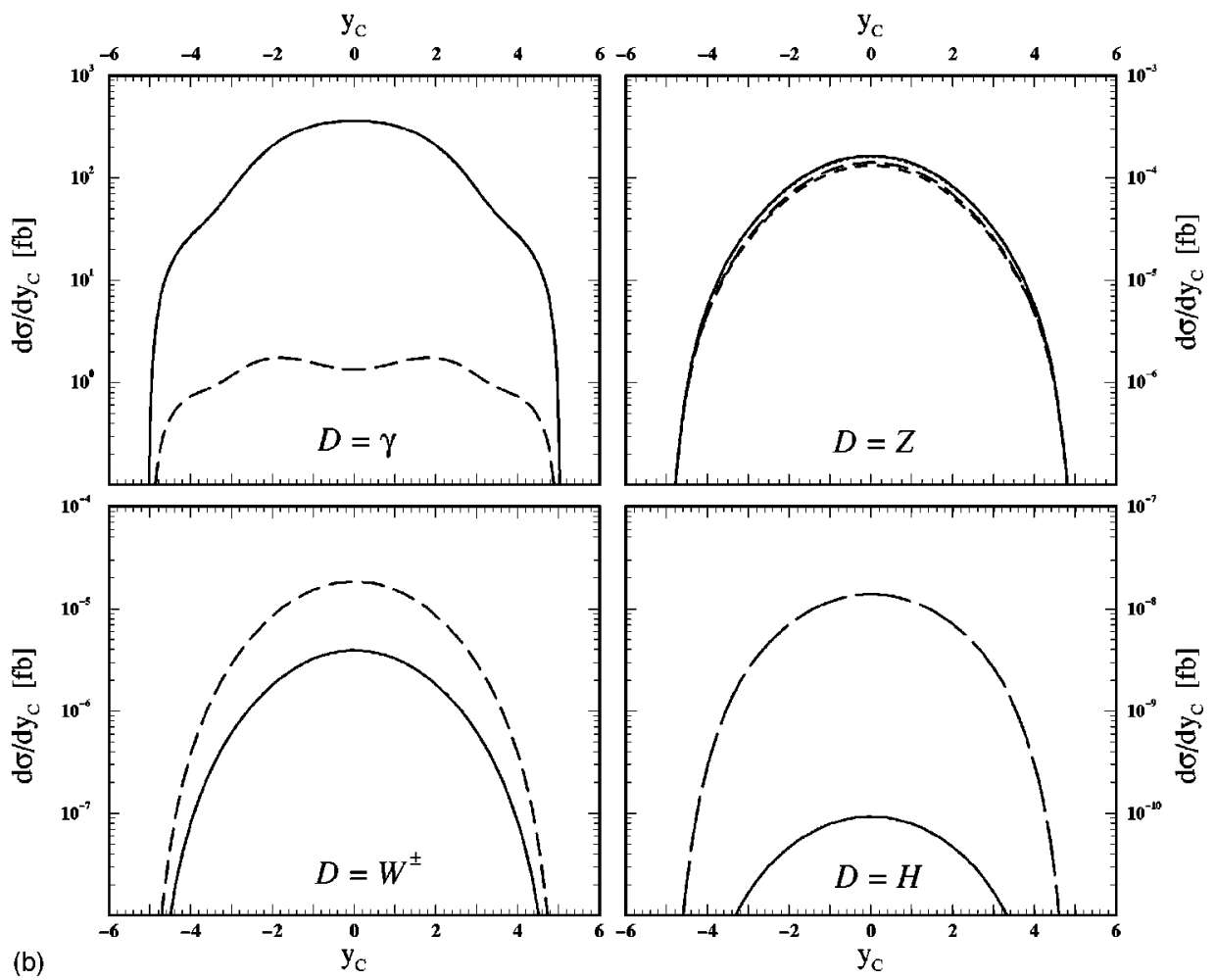

FIG. 1. (a) $p_{T}$ distributions $d \sigma / d p_{T}$ (in fb/GeV) and (b) $y_{C}$ distributions $d \sigma / d y_{C}$ (in $\mathrm{fb}$ ) of $e^{+} e^{-} \rightarrow e^{+} e^{-} C D+X$, where $C$ $=J / \psi, \chi_{c J}$ and $D=\gamma, Z, W, H$, at TESLA in the $e^{+} e^{-}$mode. It is summed over $C=\chi_{c 0}, \chi_{c 1}, \chi_{c 2}$ and $D=W^{+}, W^{-}$. In each figure, the CSM (dotted lines) and NRQCD (solid lines) predictions for $C=J / \psi$ and the CSM (shortdashed lines) and NRQCD (medium-dashed lines) ones for $C=\chi_{c J}$ are shown separately. Superposed short-dashed and medium-dashed lines appear as long-dashed lines.

(b)

the figures for $L$ in units of $10^{32} \mathrm{~cm}^{-2} \mathrm{~s}^{-1}$ presented in Table I equally correspond to the integrated luminosity $\int d t L$ in units of $\mathrm{fb}^{-1}$ per year and experiment. At the Tevatron and the LHC, the measurements can be performed simulta- neously by two dedicated experiments. We thus obtain the values of $\sigma_{1}$ specified in Table I.

We are now in a position to present our numerical results. Figures 1-5 are devoted to $e^{+} e^{-} \rightarrow e^{+} e^{-} C D+X$ in the 


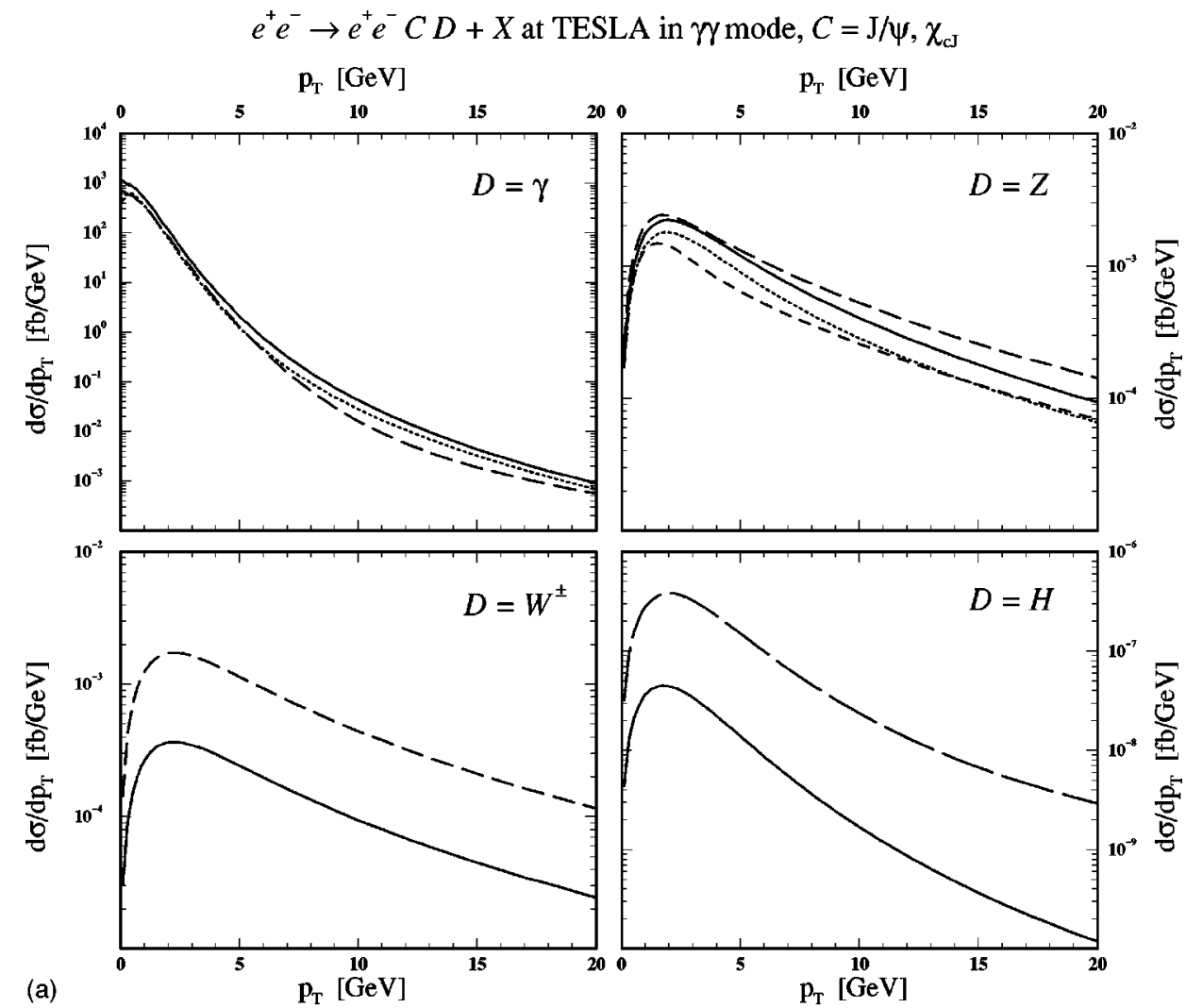

$e^{+} e^{-} \rightarrow e^{+} e^{-} C D+X$ at TESLA in $\gamma /$ mode, $C=\mathrm{J} / \psi, \chi_{\mathrm{cJ}}$

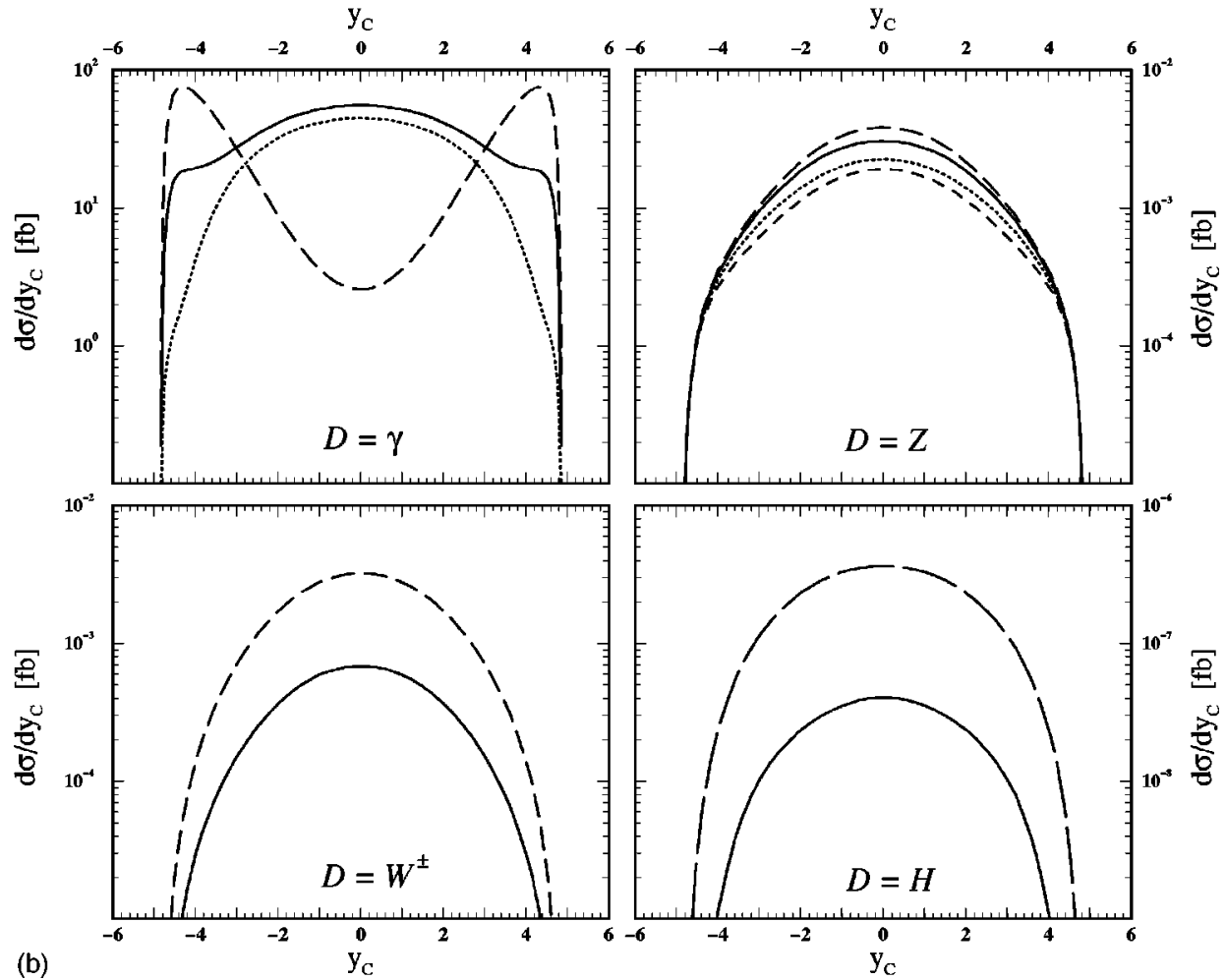

FIG. 2. Same as in Figs. 1(a) and 1(b), but for $e^{+} e^{-}$ $\rightarrow e^{+} e^{-} C D+X$ at TESLA in the $\gamma \gamma$ mode. $e^{+} e^{-}$and $\gamma \gamma$ modes of TESLA, to $p e^{ \pm} \rightarrow e^{ \pm} C D+X$ at THERA, to $p \bar{p} \rightarrow C D+X$ at the Tevatron (Run II), and to $p p \rightarrow C D+X$ at the LHC, respectively. In each figure, parts (a) and (b) give the $p_{T}$ distributions $d \sigma / d p_{T}$ and the $y_{C}$ distributions $d \sigma / d y_{C}$, respectively. In each part, there are four frames, which refer to $D=\gamma, Z, W, H$, respectively. In each frame, we separately consider $C=J / \psi, \chi_{c J}$, both in the CSM and in NRQCD. It is summed over $C=\chi_{c 0}, \chi_{c 1}, \chi_{c 2}$ 

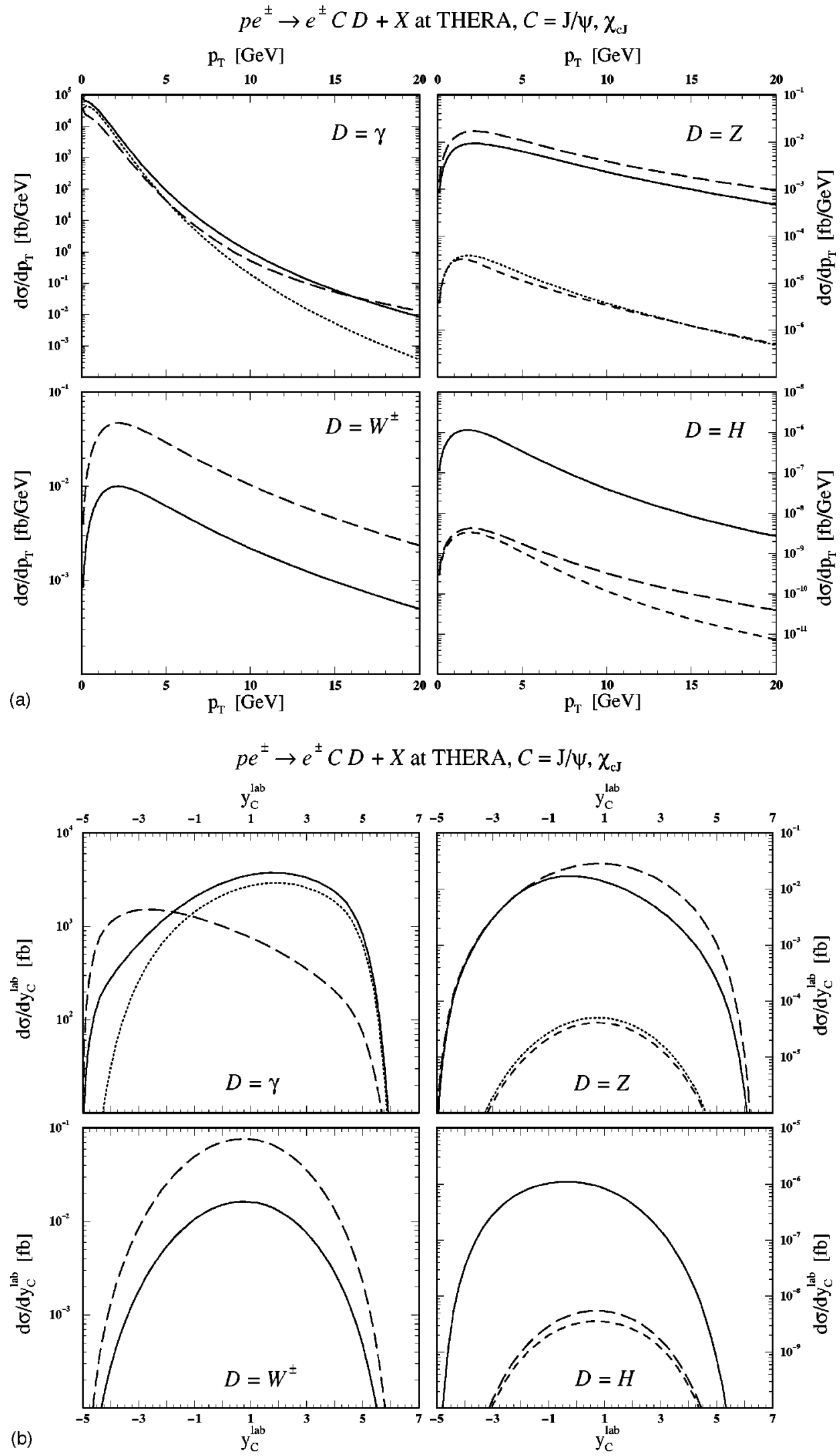

and $D=W^{+}, W^{-}$. In the case of $D=\gamma$, the $y_{C}$ distributions are evaluated imposing the cut $p_{T}>1 \mathrm{GeV}$ in order to exclude the infrared and collinear singularities mentioned inSec. II. Figure 3(b) refers to the laboratory frame, $y_{C}^{\text {lab }}$ being
FIG. 3. Same as in Figs. 1(a) and $1(\mathrm{~b})$, but for $p e^{ \pm} \rightarrow e^{ \pm} C D$ $+X$ at THERA in the laboratory frame. 

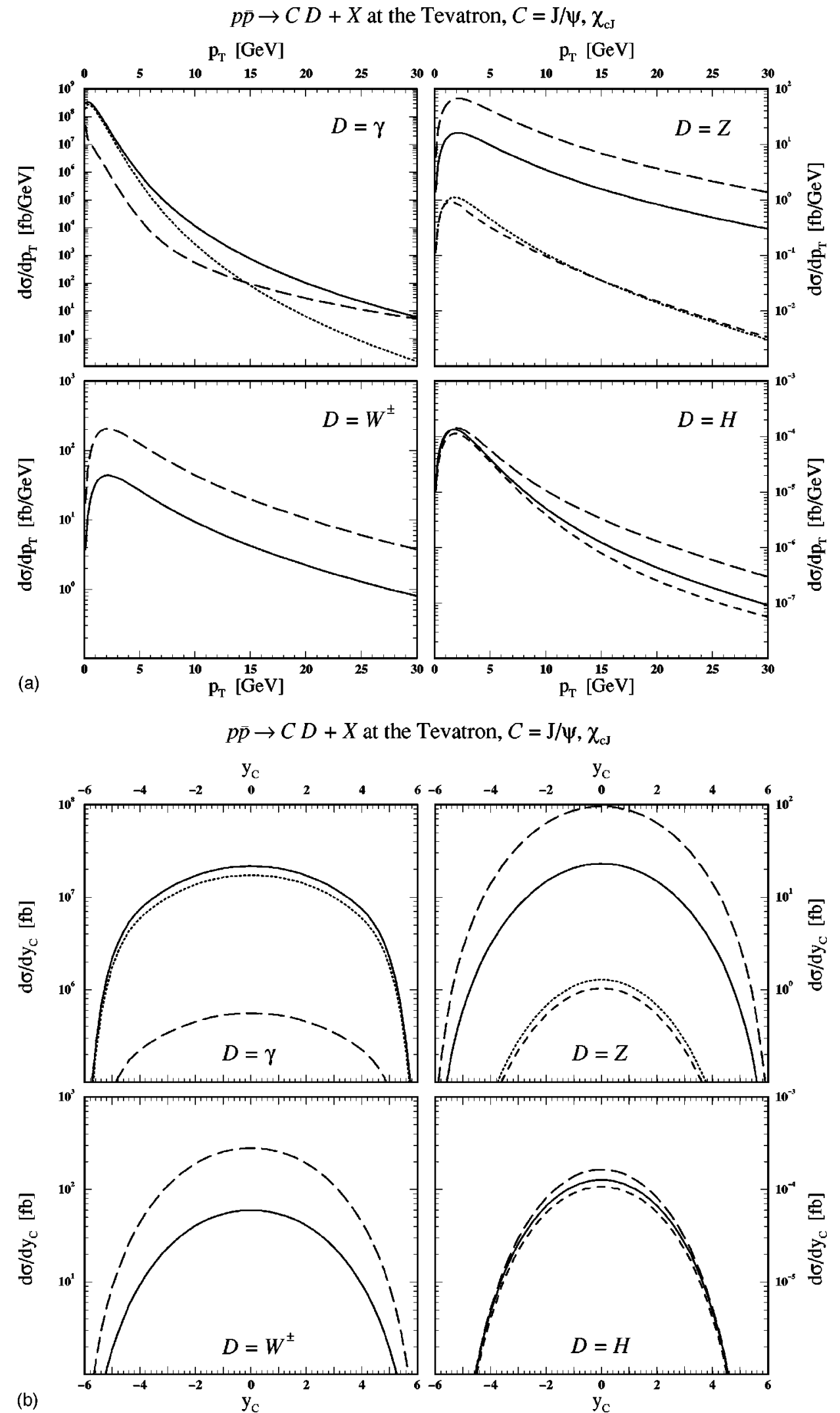

production of $\chi_{c J}+\gamma, J / \psi+W, \chi_{c J}+W$, and $J / \psi+H$ is forbidden in the CSM to the order considered.

(ii) The cross section ratio of $J / \psi+W$ and $\chi_{c J}+W$ associated production, which proceeds through processes (17)
FIG. 4. Same as in Figs. 1(a) and 1 (b), but for $p \bar{p} \rightarrow C D+X$ at the Tevatron. and (18), is always $\left.\left\langle\mathcal{O}^{J / \psi}{ }^{3} S_{1}^{(8)}\right]\right\rangle / \Sigma_{J=0}^{2}\left\langle\mathcal{O}^{\chi_{c} J}\left[{ }^{3} S_{1}^{(8)}\right]\right\rangle$ $\approx 0.21[7]$.

(iii) With increasing value of $m_{C D}$, the CO process (16) with $s={ }^{3} S_{1}$ generally gains relative importance, since its 
$p p \rightarrow C D+X$ at the $\mathrm{LHC}, C=\mathrm{J} / \psi, \chi_{\mathrm{cJ}}$

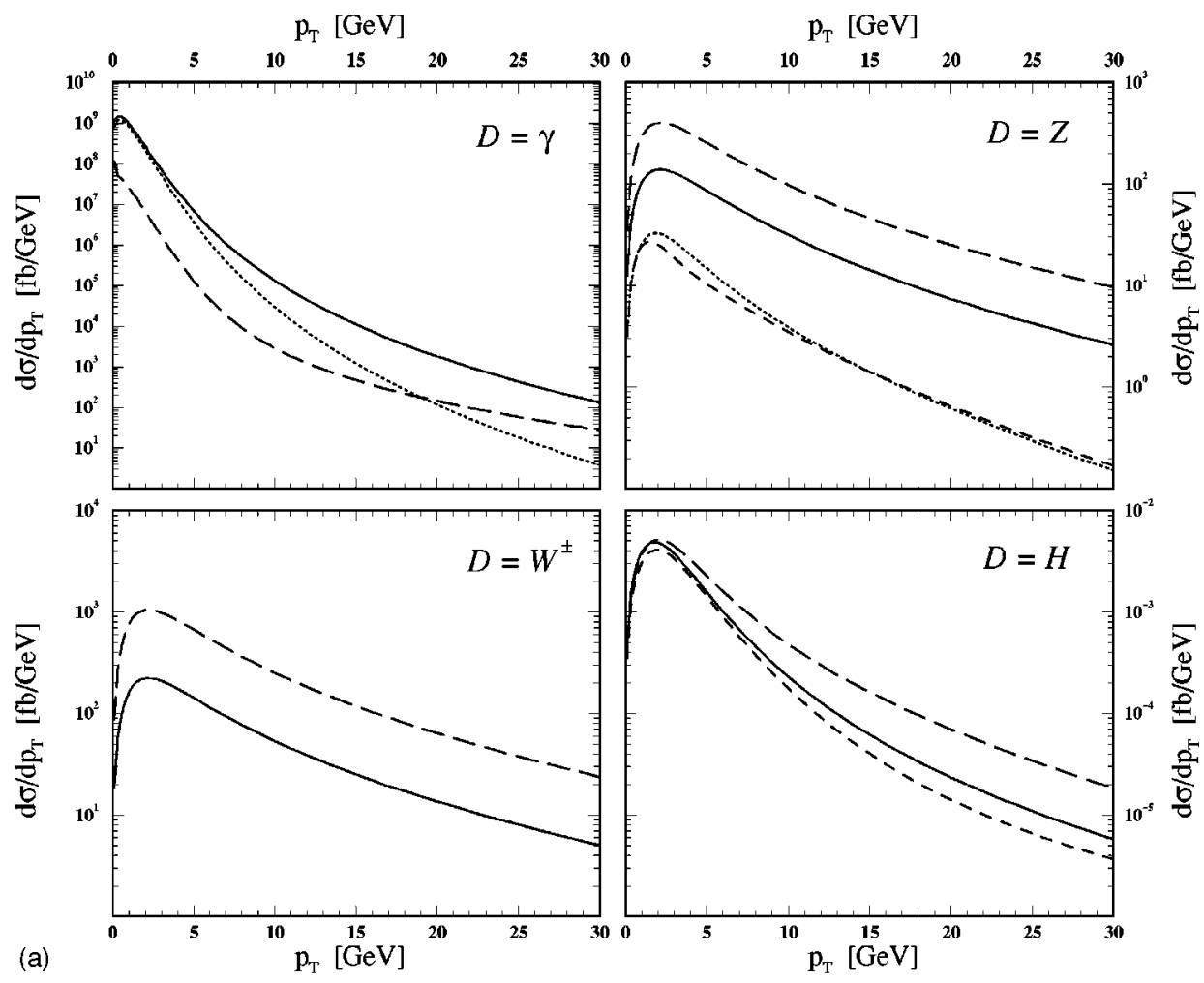

(a)

$$
p p \rightarrow C D+X \text { at the } \mathrm{LHC}, C=\mathrm{J} / \psi, \chi_{\mathrm{cJ}}
$$

FIG. 5. Same as in Figs. 1(a) and $1(\mathrm{~b})$, but for $p p \rightarrow C D+X$ at the LHC.
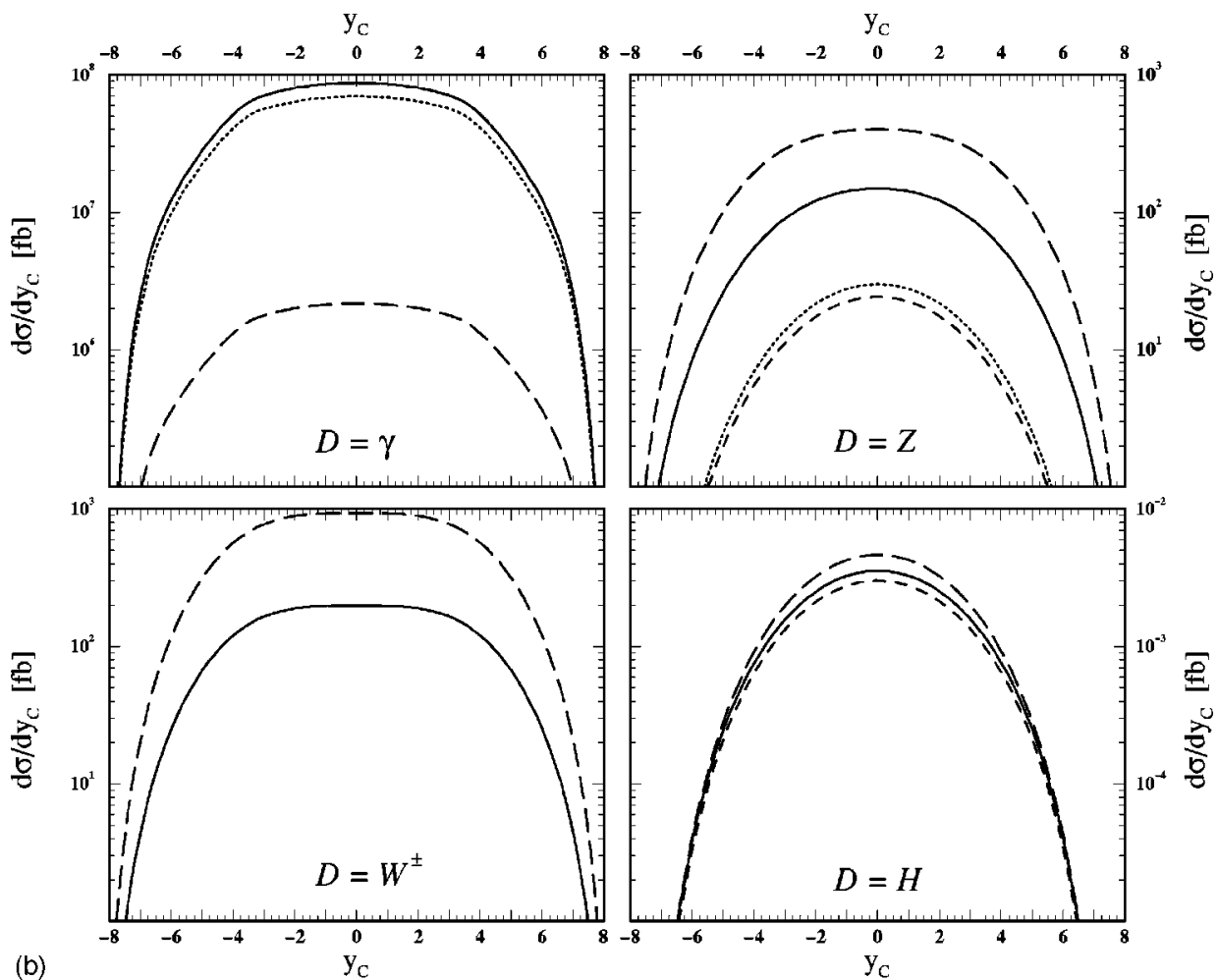

cross section involves a gluon propagator with small virtuality, $q^{2}=m_{C}^{2}$, and is, therefore, enhanced by powers of $m_{C D}^{2} / m_{C}^{2}$ relative to those of the other contributing processes. In the fragmentation picture [35], this cross section would be evaluated by convoluting the cross section of $q \bar{q} \rightarrow g N$ with the $g \rightarrow c \bar{c}\left[{ }^{3} S_{1}^{(8)}\right]$ fragmentation function [36]. Processes (17) and (18) also benefit from this type of enhancement.

(iv) The processes with $D=\gamma$ generally have much larger 
cross sections than those with $D=Z, W, H$ because the available phase space is considerably ampler and the infrared and collinear singularities at $p_{T}=0$, albeit eliminated by a minimum- $p_{T}$ cut, still feed into the finite parts of the cross sections.

(v) As mentioned in the Introduction, the cross sections of the processes with $D=H$ are suppressed relative to those with $D=Z, W$ by the smallness of the charm Yukawa coupling.

(vi) As is evident from Eqs. (12) and (13), the directphotoproduction channels in $e^{+} e^{-}$and $p e^{ \pm}$collisions correspond to pure CS and $\mathrm{CO}$ processes, respectively.

(vii) If $m_{C D} \gg m_{C}$, which is the case if $p_{T} \gg m_{C}$ or $D$ $=Z, W, H$, the resolved channels are generally suppressed against the direct ones because, according to Eq. (3), the values of $x_{a, b}$ are then close to unity, where the photon PDFs take small values.

(viii) Direct photons participate in the hard scattering with their full momenta, while resolved ones pass on only a fraction of theirs. Thus, in photoproduction at THERA, the direct and resolved cross sections are peaked in the backward and forward directions, respectively.

We now discuss the $e^{+} e^{-}$mode of TESLA [see Figs. 1(a) and $1(\mathrm{~b})]$. Here, $J / \psi+\gamma$ associated production dominantly proceeds through the direct CS process (12) with $\varsigma={ }^{3} S_{1}$ and $N=\gamma$. Consequently, in the first frames of Figs. 1(a) and $1(\mathrm{~b})$, the dotted and solid lines are superjacent. $\chi_{c J}+\gamma$ associated production only proceeds through singly resolved or doubly resolved $\mathrm{CO}$ processes and is accordingly suppressed relative to $J / \psi+\gamma$ associated production. For the reasons exposed in the preceding paragraph, the reactions with $D$ $=Z$ chiefly proceed through the direct CS processes (12) with $\varsigma={ }^{3} S_{1},{ }^{3} P_{J}$ and $N=Z$. The contributing CO processes are singly or doubly resolved and, therefore, strongly suppressed. The reactions with $D=W$ are mediated by the doubly resolved CO processes (17) and (18) and are accordingly suppressed. $\chi_{c J}+H$ associated production dominantly proceeds through direct CS processes, while the contributing $\mathrm{CO}$ processes are doubly resolved and, therefore, heavily suppressed. Consequently, in the fourth frames of Figs. 1(a) and 1(b), the short-dashed and medium-dashed lines are superjacent. On the other hand, $J / \psi+H$ associated production only proceeds through singly resolved or doubly resolved $\mathrm{CO}$ processes and is accordingly suppressed relative to $\chi_{c J}$ $+H$ associated production.

We now turn to the $\gamma \gamma$ mode of TESLA [see Figs. 2(a) and 2(b)]. In contrast to the energy spectra of bremsstrahlung and beamstrahlung, which are strongly peaked at $x=0$, the one of the back-scattered laser photons is evenly spread in the lower $x$ range and exhibits a maximum at $x=x_{\max }$. According to Eq. (2), large values of $p_{T}$ or $m_{D}$ entail large values of $x_{ \pm}$. This explains why, in the $\gamma \gamma$ mode of TESLA, the $p_{T}$ spectra are less steep and the cross sections for $D$ $=Z, W, H$ are larger than in the $e^{+} e^{-}$mode. Furthermore, the influence of the singly and doubly resolved channels is generally increased because the average photon energy is larger. Here, the CSM prediction for $J / \psi+\gamma$ associated production is dominated by the doubly resolved process (14) with $\varsigma={ }^{3} S_{1}$ and $N=\gamma$ in the lower $p_{T}$ range, for $p_{T}$ $\lesssim 5 \mathrm{GeV}$, while the direct process (12) with $\varsigma={ }^{3} S_{1}$ and $N$ $=\gamma$ preponderates for larger values of $p_{T}$. Beholding the first frame of Fig. 2(b), we observe that the $y_{C}$ distribution of $\chi_{c J}+\gamma$ associated production is peaked in the very forward and backward directions, close to the kinematic boundaries. This may be traced to the singly resolved process (13) with $\mathrm{s}={ }^{3} S_{1}$ and $N=\gamma$. This partonic subprocess also generates the pronounced shoulders in the $y_{C}$ distribution of $J / \psi+\gamma$ associated production in NRQCD, which is shown in the same figure. In the case of the $e^{+} e^{-}$mode, it is suppressed by the softness of the effective photon-energy spectrum. The increased influence of the singly resolved and doubly resolved CO processes is reflected in the first frame of Fig. 2(a) by the dispartment of the dotted and solid lines. Similar separations of CSM and NRQCD results are visible in the second frames of Figs. 2(a) and 2(b) for $D=Z$.

We now move on to THERA [see Figs. 3(a) and 3(b)]. In the CSM, $J / \psi+\gamma$ associated production now only happens in the resolved channel, through process (14) with $\mathrm{s}={ }^{3} S_{1}$ and $N=\gamma$, while, in NRQCD, it also takes place in the direct one, through process (13) with $\varsigma={ }^{3} S_{1}$ and $N=\gamma$. This explains why the dotted line in the first frame of Fig. 3(b) is peaked in the forward direction, while the solid one also exhibits a shoulder in the backward direction. The fact that this shoulder is not prominent may be understood by observing that $\left.\left\langle\mathcal{O}^{J /}{ }^{3}{ }^{3} S_{1}^{(8)}\right]\right\rangle /\left\langle\mathcal{O}^{J /} \psi\left[{ }^{3} S_{1}^{(1)}\right]\right\rangle \approx 3.4 \times 10^{-3}[7]$. Detailed inspection reveals that, contrary to naive expectations, the direct contribution is at least one order of magnitude suppressed against the resolved one, even at large values of $p_{T}$. From the same figure, we also glean that the CSM result almost exhausts the resolved contribution in the lower $p_{T}$ range, which is decisive for the $y_{C}$ distributions. In the intermediate $p_{T}$ range, $6 \lesssim p_{T} \leqslant 25 \mathrm{GeV}$, the latter is dominated by process (15) with $\varsigma={ }^{1} S_{0},{ }^{3} P_{J}$ and $N=\gamma$, while, for yet larger values of $p_{T}$, process (16) with $\varsigma={ }^{3} S_{1}$ and $N=\gamma$ gets in the lead [39]. In the case of $\chi_{c J}+\gamma$ associated production, processes (13) and (16) with $\varsigma={ }^{3} S_{1}$ and $N=\gamma$ compete with each other. The former dominates for $p_{T} \lesssim 11 \mathrm{GeV}$, while the latter prevails in the complementary $p_{T}$ range. Since the $y_{C}$ distributions reflect the situation in the small $p_{T}$ range, this explains why the medium-dashed line in the first frame of Fig. 3(b) is peaked in the backward direction. Also in the case of $J / \psi+Z$ associated production, the direct and resolved channels compete with each other. The direct one is dominated by process (13) with $\varsigma={ }^{1} S_{0},{ }^{3} P_{J}$ and $N=Z$, while the resolved one is dominated by process (16) with $s$ $={ }^{3} S_{1}$ and $N=Z$. Here, the crossover occurs at $p_{T}$ $\approx 28 \mathrm{GeV}$. By contrast, $\chi_{c J}+Z$ associated production is always overwhelmingly dominated by the very same resolved process. This explains why the solid and medium-dashed lines in the second frame of Fig. 3(b) are peaked in the backward and forward directions, respectively. In the large$p_{T}$ limit, the $J / \psi+Z$ to $\chi_{c J}+Z$ cross section ratio approaches the same value as in the case of $D=W$ discussed under item (ii) in the above enumeration. The CS processes (14) with $\varsigma={ }^{3} S_{1},{ }^{3} P_{J}$ and $N=Z$ are dramatically suppressed because they are resolved and not fragmentation prone. $J / \psi$ 
$+H$ associated production dominantly proceeds through direct $\mathrm{CO}$ processes. On the other hand, $\chi_{c J}+H$ associated production is only possible through resolved processes and is, therefore, suppressed relative to $J / \psi+H$ associated production. There are no fragmentation-prone processes in the case $D=H$ because the Higgs boson always couples to the charm-quark line.

We now proceed to the Tevatron [see Figs. 4(a) and 4(b)]. For $p_{T} \leqslant 5 \mathrm{GeV}, J / \psi+\gamma$ associated production dominantly proceeds through the CS process (14) with $\varsigma={ }^{3} S_{1}$ and $N$ $=\gamma$, due to the large value of $\left.\left\langle\mathcal{O}^{J /} \psi^{3} S_{1}^{(1)}\right]\right\rangle$. For larger values of $p_{T}$, the CO processes take over. Specifically, the latter are dominated by process (15) with $\varsigma={ }^{1} S_{0},{ }^{3} P_{J}$ and $N=\gamma$ for $p_{T} \lesssim 46 \mathrm{GeV}$, while process (16) with $\mathrm{s}={ }^{3} S_{1}$ and $N=\gamma$ prevails for larger values of $p_{T}$. On the other hand, $\chi_{c J}+\gamma$ associated production is always dominated by the latter. Similarly, in the case of $D=Z$, process (16) with s $={ }^{3} S_{1}$ and $N=Z$ is always dominant. By the same token, the $J / \psi+Z$ to $\chi_{c J}+Z$ cross section ratio is approximately the same as in the case of $D=W$ discussed above. On the other hand, the CS processes (14) with $\varsigma={ }^{3} S_{1},{ }^{3} P_{J}$ and $N=Z$ are not fragmentation prone and are, therefore, vigorously suppressed.

Finally, we arrive at the LHC [see Figs. 5(a) and 5(b)]. Here, the $p_{T}$ and $y_{C}$ distributions generally exhibit very similar shapes as in the case of the Tevatron, while their normalizations are increased by approximately the same amount as the value of $\sqrt{S}$ is.

The experimental observation of processes that are forbidden or exceedingly suppressed in the CSM with cross sections that are compatible with the NRQCD predictions would provide striking evidence for the NRQCD factorization hypothesis and firmly establish the existence of $\mathrm{CO}$ processes in nature. Furthermore, precise measurements of these cross sections would lead to useful constraints on the appearing CO MEs. On the other hand, the experimental study of processes that are dominated by CS channels would allow for independent determinations of the CS MEs, which can be compared with the results obtained using traditional methods, so as to lead to valuable consistency checks. Our analysis allows us to identify processes of all these categories. As mentioned above, the associated production of $\chi_{c J}+\gamma, J / \psi$ $+W, \chi_{c J}+W$, and $J / \psi+H$ exclusively proceeds through CO processes. At THERA, the Tevatron, and the LHC, the associated production of $J / \psi+\gamma$ and $\chi_{c J}+H$ with $p_{T} \gg m_{C}$ and of $J / \psi+Z$ and $\chi_{c J}+Z$ with arbitrary values of $p_{T}$ is greatly dominated by $\mathrm{CO}$ processes. On the other hand, examples where $\mathrm{CO}$ processes play a negligible role include the associated production of $J / \psi+\gamma, J / \psi+Z, \chi_{c J}+Z$, and $\chi_{c J}+H$ in the $e^{+} e^{-}$mode of TESLA, of $\chi_{c J}+H$ in the $\gamma \gamma$ mode of TESLA, and of $J / \psi+\gamma$ and $\chi_{c J}+H$ with small values of $p_{T}$ at THERA, the Tevatron, and the LHC.

We conclude this section by assessing the observability of the various processes in the various experiments with the aid of Table I. The processes with $D=\gamma$ will abundantly take place in all considered experiments. The processes with $D$ $=Z, W$ will produce considerable yields at the hadron colliders, namely, several hundred (ten thousand) events per year at the Tevatron (LHC), while they significantly fall short of the one-event-per-year mark at TESLA and THERA. As expected, the processes with $D=H$ are predicted to be far too rare to be observable in any of the considered experiments. In turn, their observation would hint at new physics beyond the SM.

\section{CONCLUSIONS}

We studied the associated production of heavy quarkonia $C$ with electroweak bosons $D$ in photon-photon, photonhadron, and hadron-hadron collisions to LO in the NRQCD factorization formalism. We considered all experimentally established heavy quarkonia, with ${ }^{2 S+1} L_{J}$ $={ }^{1} S_{0},{ }^{3} S_{1},{ }^{1} P_{1},{ }^{3} P_{J}$, and all electroweak bosons of the SM, $D=\gamma, Z, W, H$. We listed all contributing partonic cross sections, except for those with $D=H$, which will be irrelevant for phenomenology in the foreseeable future. We presented numerical results for any combination of $C=J / \psi, \chi_{c J}$ and $D=\gamma, Z, W, H$ appropriate for TESLA in the $e^{+} e^{-}$and $\gamma \gamma$ modes, THERA, Run II of the Tevatron, and the LHC.

At TESLA and THERA, only the processes with $D=\gamma$ will have observable cross sections, while, at the Tevatron and the LHC, this is also the case for $D=Z, W$. Observation of $\chi_{c J}+\gamma, J / \psi+W$, and $\chi_{c J}+W$ associated production, which are pure $\mathrm{CO}$ processes, would give strong support to the NRQCD factorization hypothesis and, if measured with sufficient precision, allow for independent determinations of $\left\langle\mathcal{O}^{J / \psi}\left[{ }^{3} S_{1}^{(8)}\right]\right\rangle$ and $\left\langle\mathcal{O} \chi_{c 0}\left[{ }^{3} S_{1}^{(8)}\right]\right\rangle$. A similar statement applies to $J / \psi+Z$ and $\chi_{c J}+Z$ associated production at the Tevatron and the LHC, which are overwhelmingly dominated by $\mathrm{CO}$ processes. On the other hand, $\mathrm{CO}$ processes play a subordinate role for $J / \psi+\gamma$ associated production in the $e^{+} e^{-}$mode of TESLA for arbitrary values of $p_{T}$ and at THERA, the Tevatron, and the LHC for small values of $p_{T}$. This offers the opportunity to extract a new value of $\left.\left\langle\mathcal{O}^{J /}{ }^{3}{ }^{3} S_{1}^{(1)}\right]\right\rangle$ and thus to allow for a consistency check. Alternatively, assuming $\left\langle\mathcal{O}^{J /} \psi\left[{ }^{3} S_{1}^{(1)}\right]\right\rangle$ to be sufficiently well known, one may get a better handle on the gluon PDFs of the photon and proton by fitting THERA, Tevatron, and LHC data of $J / \psi+\gamma$ associated production in the lower $p_{T}$ range.

\section{ACKNOWLEDGMENTS}

B.A.K. and C.P.P. are grateful to the CERN Theoretical Physics Division and the Second Institute for Theoretical Physics of Hamburg University, respectively, for their hospitality during visits when this paper was prepared. The research of B.A.K. was supported in part by the Deutsche Forschungsgemeinschaft through Grant No. KN 365/1-1 and by the Bundesministerium für Bildung und Forschung through Grant No. 05 HT1GUA/4. The research of C.P.P. was supported by the Office of the Vice President for Academic Affairs of the University of the Philippines. The research of L.Z. was supported by the Studienstiftung des deutschen Volkes. 


\section{APPENDIX: PARTONIC CROSS SECTIONS}

In this appendix, we list the differential cross sections $d \sigma / d t$ for processes (12)-(18) with $N=Z$ to be inserted in Eq. (1) and its counterparts for lepton-hadron and hadron-hadron scattering. The results for $N=\gamma$ are recovered as explained in Sec. II. Our results read

$$
\begin{aligned}
\frac{d \sigma}{d t}(\gamma \gamma \rightarrow & \left.Q \bar{Q}\left[{ }^{1} S_{0}^{(1)}\right] Z\right)=\frac{1024 \pi \alpha^{2} g^{2} a_{Q}^{2}\left(2 m_{Z}^{2}-s-t-u\right)^{2}}{81 M m_{Z}^{2} s^{2}\left(m_{Z}^{2}-s-t\right)^{2}\left(m_{Z}^{2}-s-u\right)^{2}\left(2 m_{Z}^{2}-t-u\right)^{2}}\left[m_{Z}^{8}-2 m_{Z}^{6}(t+u)-m_{Z}^{4}\left(s^{2}-t^{2}-4 t u-u^{2}\right)\right. \\
& \left.-2 m_{Z}^{2} t u(t+u)+t^{2} u^{2}\right]
\end{aligned}
$$

$$
\begin{aligned}
\frac{d \sigma}{d t}(\gamma \gamma \rightarrow & \left.Q \bar{Q}\left[{ }^{3} S_{1}^{(1)}\right] Z\right)=\frac{-1024 \pi \alpha^{2} g^{2} v_{Q}^{2}}{243 M s^{2}\left(m_{Z}^{2}-s-t\right)^{2}\left(m_{Z}^{2}-s-u\right)^{2}\left(2 m_{Z}^{2}-t-u\right)^{2}}\left\{m_{Z}^{10}-4 m_{Z}^{8}(3 s+t+u)+m_{Z}^{6}\left[22 s^{2}+26 s(t+u)\right.\right. \\
& \left.+5 t^{2}+12 t u+5 u^{2}\right]-2 m_{Z}^{4}\left[5 s^{3}+14 s^{2}(t+u)+s\left(8 t^{2}+23 t u+8 u^{2}\right)+(t+u)\left(t^{2}+5 t u+u^{2}\right)\right]-m_{Z}^{2}\left[s^{4}-4 s^{3}(t\right. \\
& \left.+u)-s^{2}\left(9 t^{2}+26 t u+9 u^{2}\right)-2 s(t+u)\left(t^{2}+10 t u+u^{2}\right)-t u\left(4 t^{2}+9 t u+4 u^{2}\right)\right]-2\left[s^{3}\left(t^{2}+t u+u^{2}\right)+s^{2}(t+u)^{3}\right. \\
& \left.\left.+s t u\left(t^{2}+3 t u+u^{2}\right)+t^{2} u^{2}(t+u)\right]\right\}
\end{aligned}
$$

$$
\begin{aligned}
& \frac{d \sigma}{d t}\left(\gamma \gamma \rightarrow Q \bar{Q}\left[{ }^{1} P_{1}^{(1)}\right] Z\right)=\frac{-8192 \pi \alpha^{2} g^{2} v_{Q}^{2}}{243 M^{3} s^{2}\left(m_{Z}^{2}-s-t\right)^{3}\left(m_{Z}^{2}-s-u\right)^{3}\left(2 m_{Z}^{2}-t-u\right)^{4}}\left\{48 m_{Z}^{18}-16 m_{Z}^{16}[11 s+15(t+u)]+2 m_{Z}^{14}\left[141 s^{2}\right.\right. \\
& \left.+374 s(t+u)+4\left(65 t^{2}+134 t u+65 u^{2}\right)\right]-4 m_{Z}^{12}\left[65 s^{3}+255 s^{2}(t+u)+s\left(341 t^{2}+696 t u+341 u^{2}\right)+32(t+u)\right. \\
& \left.\times\left(5 t^{2}+11 t u+5 u^{2}\right)\right]+m_{Z}^{10}\left[138 s^{4}+798 s^{3}(t+u)+10 s^{2}\left(158 t^{2}+315 t u+158 u^{2}\right)+s(t+u)\left(1399 t^{2}+2958 t u\right.\right. \\
& \left.\left.+1399 u^{2}\right)+491 t^{4}+2188 t^{3} u+3378 t^{2} u^{2}+2188 t u^{3}+491 u^{4}\right]-m_{Z}^{8}\left[24 s^{5}+356 s^{4}(t+u)+20 s^{3}\left(53 t^{2}+101 t u+53 u^{2}\right)\right. \\
& +10 s^{2}(t+u)\left(138 t^{2}+265 t u+138 u^{2}\right)+s(t+u)^{2}\left(887 t^{2}+1956 t u+887 u^{2}\right)+(t+u)\left(241 t^{4}+1188 t^{3} u+1846 t^{2} u^{2}\right. \\
& \left.\left.+1188 t u^{3}+241 u^{4}\right)\right]-m_{Z}^{6}\left[18 s^{6}-60 s^{5}(t+u)-s^{4}\left(417 t^{2}+734 t u+417 u^{2}\right)-s^{3}(t+u)\left(801 t^{2}+1298 t u+801 u^{2}\right)\right. \\
& -s^{2}\left(749 t^{4}+2772 t^{3} u+4068 t^{2} u^{2}+2772 t u^{3}+749 u^{4}\right)-s(t+u)\left(359 t^{4}+1534 t^{3} u+2362 t^{2} u^{2}+1534 t u^{3}+359 u^{4}\right) \\
& \left.-2(t+u)^{2}\left(37 t^{4}+217 t^{3} u+332 t^{2} u^{2}+217 t u^{3}+37 u^{4}\right)\right]+m_{Z}^{4}\left[12 s^{7}+16 s^{6}(t+u)-s^{5}\left(83 t^{2}+122 t u+83 u^{2}\right)\right. \\
& -s^{4}(t+u)\left(275 t^{2}+356 t u+275 u^{2}\right)-s^{3}\left(367 t^{4}+1153 t^{3} u+1580 t^{2} u^{2}+1153 t u^{3}+367 u^{4}\right)-s^{2}(t+u)\left(258 t^{4}+843 t^{3} u\right. \\
& \left.+1214 t^{2} u^{2}+843 t u^{3}+258 u^{4}\right)-4 s\left(23 t^{6}+143 t^{5} u+371 t^{4} u^{2}+500 t^{3} u^{3}+371 t^{2} u^{4}+143 t u^{5}+23 u^{6}\right)-(t+u)^{3} \\
& \left.\times\left(13 t^{4}+104 t^{3} u+150 t^{2} u^{2}+104 t u^{3}+13 u^{4}\right)\right]-m_{Z}^{2}\left[2 s^{8}+6 s^{7}(t+u)-s^{6}\left(7 t^{2}+4 t u+7 u^{2}\right)-52 s^{5}(t+u)\right. \\
& \times\left(t^{2}+t u+u^{2}\right)-s^{4}\left(97 t^{4}+269 t^{3} u+346 t^{2} u^{2}+269 t u^{3}+97 u^{4}\right)-2 s^{3}(t+u)\left(48 t^{4}+122 t^{3} u+155 t^{2} u^{2}\right. \\
& \left.+122 t u^{3}+48 u^{4}\right)-s^{2}\left(53 t^{6}+244 t^{5} u+531 t^{4} u^{2}+674 t^{3} u^{3}+531 t^{2} u^{4}+244 t u^{5}+53 u^{6}\right)-s(t+u)\left(14 t^{6}+82 t^{5} u\right. \\
& \left.\left.+217 t^{4} u^{2}+290 t^{3} u^{3}+217 t^{2} u^{4}+82 t u^{5}+14 u^{6}\right)-(t+u)^{4}\left(t^{4}+15 t^{3} u+19 t^{2} u^{2}+15 t u^{3}+u^{4}\right)\right]-(t+u)\left[s^{7}(t+u)\right. \\
& +s^{6}\left(5 t^{2}+6 t u+5 u^{2}\right)+s^{5}(t+u)\left(11 t^{2}+8 t u+11 u^{2}\right)+2 s^{4}\left(7 t^{4}+16 t^{3} u+19 t^{2} u^{2}+16 t u^{3}+7 u^{4}\right)+s^{3}(t+u) \\
& \times\left(11 t^{4}+21 t^{3} u+25 t^{2} u^{2}+21 t u^{3}+11 u^{4}\right)+s^{2}\left(5 t^{6}+19 t^{5} u+38 t^{4} u^{2}+46 t^{3} u^{3}+38 t^{2} u^{4}+19 t u^{5}+5 u^{6}\right)+s(t+u) \\
& \left.\left.\times\left(t^{6}+5 t^{5} u+14 t^{4} u^{2}+18 t^{3} u^{3}+14 t^{2} u^{4}+5 t u^{5}+u^{6}\right)+t u(t+u)^{4}\left(t^{2}+t u+u^{2}\right)\right]\right\}, \\
& \frac{d \sigma}{d t}\left(\gamma \gamma \rightarrow Q \bar{Q}\left[{ }^{3} P_{J}^{(1)}\right] Z\right)=\frac{2048 \pi \alpha^{2} g^{2} a_{Q}^{2}}{1215 M^{3} m_{Z}^{2} s^{2}\left(m_{Z}^{2}-s-t\right)^{4}\left(m_{Z}^{2}-s-u\right)^{4}\left(2 m_{Z}^{2}-t-u\right)^{4}} F_{J}, \quad J=0,1,2
\end{aligned}
$$




$$
\begin{aligned}
& F_{0}=10\left(2 m_{Z}^{2}-s-t-u\right)^{2}\left\{4 m_{Z}^{18} s-4 m_{Z}^{16}\left[2 s^{2}+4 s(t+u)-(t-u)^{2}\right]-2 m_{Z}^{14}\left[10 s^{3}-s\left(9 t^{2}+38 t u+9 u^{2}\right)+10(t+u)(t-u)^{2}\right]\right. \\
& +m_{Z}^{12}\left[80 s^{4}+176 s^{3}(t+u)+2 s^{2}\left(35 t^{2}+46 t u+35 u^{2}\right)+2 s(t+u)\left(3 t^{2}-62 t u+3 u^{2}\right)+(t-u)^{2}\left(41 t^{2}+90 t u+41 u^{2}\right)\right] \\
& -2 m_{Z}^{10}\left[50 s^{5}+192 s^{4}(t+u)+8 s^{3}\left(27 t^{2}+50 t u+27 u^{2}\right)+2 s^{2}(t+u)\left(35 t^{2}+48 t u+35 u^{2}\right)+s\left(13 t^{4}-23 t^{3} u-120 t^{2} u^{2}\right.\right. \\
& \left.\left.-23 t u^{3}+13 u^{4}\right)+2(t-u)^{2}(t+u)\left(11 t^{2}+30 t u+11 u^{2}\right)\right]+m_{Z}^{8}\left[56 s^{6}+336 s^{5}(t+u)+64 s^{4}\left(10 t^{2}+19 t u+10 u^{2}\right)\right. \\
& +4 s^{3}(t+u)\left(123 t^{2}+220 t u+123 u^{2}\right)+s^{2}\left(125 t^{4}+476 t^{3} u+598 t^{2} u^{2}+476 t u^{3}+125 u^{4}\right)+2 s(t+u)\left(9 t^{4}+18 t^{3} u\right. \\
& \left.\left.-110 t^{2} u^{2}+18 t u^{3}+9 u^{4}\right)+2(t-u)^{2}\left(13 t^{4}+77 t^{3} u+130 t^{2} u^{2}+77 t u^{3}+13 u^{4}\right)\right]-2 m_{Z}^{6}\left[6 s^{7}+64 s^{6}(t+u)+s^{5}\right. \\
& \times\left(191 t^{2}+370 t u+191 u^{2}\right)+2 s^{4}(t+u)\left(127 t^{2}+232 t u+127 u^{2}\right)+s^{3}\left(157 t^{4}+582 t^{3} u+828 t^{2} u^{2}+582 t u^{3}+157 u^{4}\right) \\
& +2 s^{2}(t+u)\left(17 t^{4}+70 t^{3} u+70 t^{2} u^{2}+70 t u^{3}+17 u^{4}\right)+s\left(2 t^{6}+30 t^{5} u-9 t^{4} u^{2}-102 t^{3} u^{3}-9 t^{2} u^{4}+30 t u^{5}+2 u^{6}\right) \\
& \left.+(t-u)^{2}(t+u)\left(4 t^{4}+35 t^{3} u+68 t^{2} u^{2}+35 t u^{3}+4 u^{4}\right)\right]+m_{Z}^{4}\left[16 s^{7}(t+u)+86 s^{6}(t+u)^{2}+2 s^{5}(t+u)\left(95 t^{2}+182 t u+95 u^{2}\right)\right. \\
& +s^{4}(t+u)^{2}\left(217 t^{2}+358 t u+217 u^{2}\right)+2 s^{3}(t+u)\left(63 t^{4}+204 t^{3} u+278 t^{2} u^{2}+204 t u^{3}+63 u^{4}\right)+2 s^{2}\left(14 t^{6}+71 t^{5} u+145 t^{4} u^{2}\right. \\
& \left.+160 t^{3} u^{3}+145 t^{2} u^{4}+71 t u^{5}+14 u^{6}\right)+2 s t u(t+u)\left(10 t^{4}+11 t^{3} u-50 t^{2} u^{2}+11 t u^{3}+10 u^{4}\right)+(t-u)^{2}(t+u)^{2}\left(t^{4}+18 t^{3} u\right. \\
& \left.\left.+47 t^{2} u^{2}+18 t u^{3}+u^{4}\right)\right]-2 m_{Z}^{2}\left[2 s^{7}(t+u)^{2}+10 s^{6}(t+u)^{3}+s^{5}\left(22 t^{4}+81 t^{3} u+120 t^{2} u^{2}+81 t u^{3}+22 u^{4}\right)\right. \\
& +2 s^{4}(t+u)\left(13 t^{4}+40 t^{3} u+56 t^{2} u^{2}+40 t u^{3}+13 u^{4}\right)+4 s^{3}\left(t^{2}+t u+u^{2}\right)^{2}\left(4 t^{2}+9 t u+4 u^{2}\right)+s^{2}(t+u)\left(4 t^{6}+13 t^{5} u\right. \\
& \left.+26 t^{4} u^{2}+22 t^{3} u^{3}+26 t^{2} u^{4}+13 t u^{5}+4 u^{6}\right)+s t u\left(t^{6}+9 t^{5} u-2 t^{4} u^{2}-18 t^{3} u^{3}-2 t^{2} u^{4}+9 t u^{5}+u^{6}\right)+t u(t-u)^{2}(t+u)^{3}\left(t^{2}\right. \\
& \left.\left.\left.+5 t u+u^{2}\right)\right]+(s+t+u)^{2}(t+u)^{2}\left[s^{4}(t+u)^{2}+2 s^{3}(t+u)\left(t^{2}+u^{2}\right)+s^{2}\left(t^{2}+u^{2}\right)^{2}+t^{2} u^{2}(t-u)^{2}\right]\right\},
\end{aligned}
$$

$F_{1}=-5\left\{176 m_{Z}^{24}-16 m_{Z}^{22}[64 s+63(t+u)]+12 m_{Z}^{20}\left[208 s^{2}+406 s(t+u)+203 t^{2}+446 t u+203 u^{2}\right]-4 m_{Z}^{18}\left[864 s^{3}+2341 s^{2}\right.\right.$

$\left.\times(t+u)+4 s\left(573 t^{2}+1315 t u+573 u^{2}\right)+2(t+u)\left(397 t^{2}+1086 t u+397 u^{2}\right)\right]+2 m_{Z}^{16}\left[1700 s^{4}+4810 s^{3}(t+u)\right.$ $+2 s^{2}\left(2969 t^{2}+7482 t u+2969 u^{2}\right)+s(t+u)\left(3947 t^{2}+13458 t u+3947 u^{2}\right)+1157 t^{4}+6964 t^{3} u+11838 t^{2} u^{2}+6964 t u^{3}$ $\left.+1157 u^{4}\right]-4 m_{Z}^{14}\left[764 s^{5}+1843 s^{4}(t+u)+s^{3}\left(1409 t^{2}+4728 t u+1409 u^{2}\right)+19 s^{2}(t+u)\left(25 t^{2}+324 t u+25 u^{2}\right)\right.$ $\left.+s\left(341 t^{4}+5925 t^{3} u+11740 t^{2} u^{2}+5925 t u^{3}+341 u^{4}\right)+3(t+u)\left(65 t^{4}+664 t^{3} u+1350 t^{2} u^{2}+664 t u^{3}+65 u^{4}\right)\right]$ $+m_{Z}^{12}\left[2552 s^{6}+6668 s^{5}(t+u)+4 s^{4}\left(467 t^{2}+2408 t u+467 u^{2}\right)-2 s^{3}(t+u)\left(4693 t^{2}-714 t u+4693 u^{2}\right)-s^{2}\left(10469 t^{4}\right.\right.$ $\left.+15252 t^{3} u+5214 t^{2} u^{2}+15252 t u^{3}+10469 u^{4}\right)-2 s(t+u)\left(1719 t^{4}-461 t^{3} u-8172 t^{2} u^{2}-461 t u^{3}+1719 u^{4}\right)-95 t^{6}$ $\left.+2048 t^{5} u+11983 t^{4} u^{2}+19840 t^{3} u^{3}+11983 t^{2} u^{4}+2048 t u^{5}-95 u^{6}\right]-2 m_{Z}^{10}\left[784 s^{7}+2838 s^{6}(t+u)+2 s^{5}\left(1149 t^{2}+3098 t u\right.\right.$ $\left.+1149 u^{2}\right)-2 s^{4}(t+u)\left(1943 t^{2}+1008 t u+1943 u^{2}\right)-s^{3}\left(8747 t^{4}+25789 t^{3} u+31940 t^{2} u^{2}+25789 t u^{3}+8747 u^{4}\right)$ $-s^{2}(t+u)\left(6261 t^{4}+17595 t^{3} u+16888 t^{2} u^{2}+17595 t u^{3}+6261 u^{4}\right)-s\left(1741 t^{6}+7835 t^{5} u+10749 t^{4} u^{2}+8966 t^{3} u^{3}\right.$ $\left.\left.+10749 t^{2} u^{4}+7835 t u^{5}+1741 u^{6}\right)-(t+u)\left(99 t^{6}+343 t^{5} u-679 t^{4} u^{2}-2118 t^{3} u^{3}-679 t^{2} u^{4}+343 t u^{5}+99 u^{6}\right)\right]+m_{Z}^{8}\left[584 s^{8}\right.$ $+2956 s^{7}(t+u)+4 s^{6}\left(1259 t^{2}+2842 t u+1259 u^{2}\right)+2 s^{5}(t+u)\left(237 t^{2}+2626 t u+237 u^{2}\right)-s^{4}\left(9273 t^{4}+30930 t^{3} u\right.$ $\left.+40826 t^{2} u^{2}+30930 t u^{3}+9273 u^{4}\right)-2 s^{3}(t+u)\left(6289 t^{4}+22639 t^{3} u+28320 t^{2} u^{2}+22639 t u^{3}+6289 u^{4}\right)-s^{2}\left(7039 t^{6}\right.$ $\left.+40430 t^{5} u+86805 t^{4} u^{2}+105964 t^{3} u^{3}+86805 t^{2} u^{4}+40430 t u^{5}+7039 u^{6}\right)-2 s(t+u)\left(811 t^{6}+4992 t^{5} u+9574 t^{4} u^{2}\right.$ $\left.+9982 t^{3} u^{3}+9574 t^{2} u^{4}+4992 t u^{5}+811 u^{6}\right)-2\left(39 t^{8}+404 t^{7} u+1209 t^{6} u^{2}+1488 t^{5} u^{3}+1280 t^{4} u^{4}+1488 t^{3} u^{5}+1209 t^{2} u^{6}\right.$ $\left.\left.+404 t u^{7}+39 u^{8}\right)\right]-2 m_{Z}^{6}\left[56 s^{9}+386 s^{8}(t+u)+2 s^{7}\left(539 t^{2}+1164 t u+539 u^{2}\right)+2 s^{6}(t+u)\left(595 t^{2}+1466 t u+595 u^{2}\right)\right.$ $-2 s^{5}\left(201 t^{4}+599 t^{3} u+588 t^{2} u^{2}+599 t u^{3}+201 u^{4}\right)-2 s^{4}(t+u)\left(1189 t^{4}+4965 t^{3} u+6653 t^{2} u^{2}+4965 t u^{3}+1189 u^{4}\right)$ $-s^{3}\left(2455 t^{6}+15774 t^{5} u+37813 t^{4} u^{2}+48736 t^{3} u^{3}+37813 t^{2} u^{4}+15774 t u^{5}+2455 u^{6}\right)-s^{2}(t+u)\left(1127 t^{6}+7744 t^{5} u\right.$ $\left.+18069 t^{4} u^{2}+22220 t^{3} u^{3}+18069 t^{2} u^{4}+7744 t u^{5}+1127 u^{6}\right)-s\left(213 t^{8}+2150 t^{7} u+7654 t^{6} u^{2}+14044 t^{5} u^{3}+16614 t^{4} u^{4}\right.$ $\left.+14044 t^{3} u^{5}+7654 t^{2} u^{6}+2150 t u^{7}+213 u^{8}\right)-(t+u)\left(7 t^{8}+118 t^{7} u+528 t^{6} u^{2}+978 t^{5} u^{3}+1098 t^{4} u^{4}+978 t^{3} u^{5}\right.$ 
$\left.\left.+528 t^{2} u^{6}+118 t u^{7}+7 u^{8}\right)\right]+m_{Z}^{4}\left[8 s^{10}+76 s^{9}(t+u)+8 s^{8}\left(44 t^{2}+93 t u+44 u^{2}\right)+6 s^{7}(t+u)\left(127 t^{2}+278 t u+127 u^{2}\right)\right.$ $+s^{6}\left(597 t^{4}+2324 t^{3} u+3574 t^{2} u^{2}+2324 t u^{3}+597 u^{4}\right)-2 s^{5}(t+u)\left(233 t^{4}+1377 t^{3} u+1950 t^{2} u^{2}+1377 t u^{3}+233 u^{4}\right)$ $-s^{4}\left(1327 t^{6}+9644 t^{5} u+25033 t^{4} u^{2}+33280 t^{3} u^{3}+25033 t^{2} u^{4}+9644 t u^{5}+1327 u^{6}\right)-2 s^{3}(t+u)\left(547 t^{6}+4136 t^{5} u\right.$ $\left.+10580 t^{4} u^{2}+13738 t^{3} u^{3}+10580 t^{2} u^{4}+4136 t u^{5}+547 u^{6}\right)-s^{2}\left(413 t^{8}+4348 t^{7} u+16425 t^{6} u^{2}+32576 t^{5} u^{3}+40108 t^{4} u^{4}\right.$ $\left.+32576 t^{3} u^{5}+16425 t^{2} u^{6}+4348 t u^{7}+413 u^{8}\right)-2 s(t+u)\left(31 t^{8}+409 t^{7} u+1674 t^{6} u^{2}+3332 t^{5} u^{3}+4016 t^{4} u^{4}+3332 t^{3} u^{5}\right.$ $\left.\left.+1674 t^{2} u^{6}+409 t u^{7}+31 u^{8}\right)-(t+u)^{2}\left(t^{8}+34 t^{7} u+225 t^{6} u^{2}+524 t^{5} u^{3}+628 t^{4} u^{4}+524 t^{3} u^{5}+225 t^{2} u^{6}+34 t u^{7}+u^{8}\right)\right]$ $-2 m_{Z}^{2}\left[6 s^{9}(t+u)^{2}+30 s^{8}(t+u)^{3}+s^{7}(t+u)^{2}\left(51 t^{2}+83 t u+51 u^{2}\right)+s^{6}(t+u)\left(13 t^{4}-39 t^{3} u-98 t^{2} u^{2}-39 t u^{3}+13 u^{4}\right)\right.$ $-s^{5}\left(70 t^{6}+613 t^{5} u+1782 t^{4} u^{2}+2470 t^{3} u^{3}+1782 t^{2} u^{4}+613 t u^{5}+70 u^{6}\right)-s^{4}(t+u)\left(104 t^{6}+873 t^{5} u+2434 t^{4} u^{2}\right.$ $\left.+3298 t^{3} u^{3}+2434 t^{2} u^{4}+873 t u^{5}+104 u^{6}\right)-s^{3}\left(65 t^{8}+733 t^{7} u+2918 t^{6} u^{2}+6080 t^{5} u^{3}+7652 t^{4} u^{4}+6080 t^{3} u^{5}\right.$ $\left.+2918 t^{2} u^{6}+733 t u^{7}+65 u^{8}\right)-s^{2}(t+u)\left(19 t^{8}+263 t^{7} u+1082 t^{6} u^{2}+2252 t^{5} u^{3}+2802 t^{4} u^{4}+2252 t^{3} u^{5}+1082 t^{2} u^{6}\right.$ $\left.+263 t u^{7}+19 u^{8}\right)-s(t+u)^{2}\left(2 t^{8}+43 t^{7} u+200 t^{6} u^{2}+440 t^{5} u^{3}+540 t^{4} u^{4}+440 t^{3} u^{5}+200 t^{2} u^{6}+43 t u^{7}+2 u^{8}\right)$ $\left.-t u(t+u)^{3}\left(t^{2}+t u+u^{2}\right)\left(t^{4}+11 t^{3} u+24 t^{2} u^{2}+11 t u^{3}+u^{4}\right)\right]-(t+u)(s+t+u)^{2}\left[s^{6}(t+u)\left(t^{2}+4 t u+u^{2}\right)\right.$ $+2 s^{5}\left(2 t^{4}+11 t^{3} u+20 t^{2} u^{2}+11 t u^{3}+2 u^{4}\right)+2 s^{4}(t+u)\left(3 t^{4}+17 t^{3} u+32 t^{2} u^{2}+17 t u^{3}+3 u^{4}\right)+2 s^{3}(t+u)^{2}$ $\times\left(2 t^{4}+15 t^{3} u+24 t^{2} u^{2}+15 t u^{3}+2 u^{4}\right)+s^{2}(t+u)\left(t^{6}+18 t^{5} u+48 t^{4} u^{2}+68 t^{3} u^{3}+48 t^{2} u^{4}+18 t u^{5}+u^{6}\right)$ $\left.\left.+2 s t u\left(t^{2}+t u+u^{2}\right)\left(2 t^{4}+7 t^{3} u+12 t^{2} u^{2}+7 t u^{3}+2 u^{4}\right)+t^{2} u^{2}(t+u)^{5}\right]\right\}$,

$F_{2}=96 m_{Z}^{24}-64 m_{Z}^{22}[11 s+15(t+u)]+8 m_{Z}^{20}\left[192 s^{2}+713 s(t+u)+28\left(17 t^{2}+35 t u+17 u^{2}\right)\right]+4 m_{Z}^{18}\left[40 s^{3}-2689 s^{2}(t+u)\right.$ $\left.-2 s\left(2359 t^{2}+4960 t u+2359 u^{2}\right)-12(t+u)\left(173 t^{2}+384 t u+173 u^{2}\right)\right]-2 m_{Z}^{16}\left[3020 s^{4}-610 s^{3}(t+u)-2 s^{2}\left(7615 t^{2}\right.\right.$ $\left.\left.+16398 t u+7615 u^{2}\right)-3 s(t+u)\left(5775 t^{2}+13402 t u+5775 u^{2}\right)-5647 t^{4}-25540 t^{3} u-39866 t^{2} u^{2}-25540 t u^{3}-5647 u^{4}\right]$ $+4 m_{Z}^{14}\left[2836 s^{5}+5845 s^{4}(t+u)-2 s^{3}\left(730 t^{2}+1927 t u+730 u^{2}\right)-2 s^{2}(t+u)\left(5965 t^{2}+14477 t u+5965 u^{2}\right)-s\left(9956 t^{4}\right.\right.$ $\left.\left.+46445 t^{3} u+73390 t^{2} u^{2}+46445 t u^{3}+9956 u^{4}\right)-2(t+u)\left(1269 t^{4}+6380 t^{3} u+10334 t^{2} u^{2}+6380 t u^{3}+1269 u^{4}\right)\right]$ $-m_{Z}^{12}\left[10600 s^{6}+36980 s^{5}(t+u)+4 s^{4}\left(9559 t^{2}+19444 t u+9559 u^{2}\right)-2 s^{3}(t+u)\left(5559 t^{2}+16706 t u+5559 u^{2}\right)\right.$ $-s^{2}\left(47103 t^{4}+224004 t^{3} u+357674 t^{2} u^{2}+224004 t u^{3}+47103 u^{4}\right)-2 s(t+u)\left(15175 t^{4}+78107 t^{3} u+129108 t^{2} u^{2}\right.$ $\left.\left.+78107 t u^{3}+15175 u^{4}\right)-6165 t^{6}-48348 t^{5} u-139987 t^{4} u^{2}-195640 t^{3} u^{3}-139987 t^{2} u^{4}-48348 t u^{5}-6165 u^{6}\right]$ $+2 m_{Z}^{10}\left[2880 s^{7}+13554 s^{6}(t+u)+4 s^{5}\left(6177 t^{2}+12874 t u+6177 u^{2}\right)+2 s^{4}(t+u)\left(8249 t^{2}+18092 t u+8249 u^{2}\right)\right.$ $-s^{3}\left(6683 t^{4}+31527 t^{3} u+51696 t^{2} u^{2}+31527 t u^{3}+6683 u^{4}\right)-s^{2}(t+u)\left(15723 t^{4}+78965 t^{3} u+132576 t^{2} u^{2}+78965 t u^{3}\right.$ $\left.+15723 u^{4}\right)-3 s\left(2621 t^{6}+20411 t^{5} u+59773 t^{4} u^{2}+84038 t^{3} u^{3}+59773 t^{2} u^{4}+20411 t u^{5}+2621 u^{6}\right)-(t+u)\left(1257 t^{6}\right.$ $\left.\left.+11553 t^{5} u+36251 t^{4} u^{2}+51926 t^{3} u^{3}+36251 t^{2} u^{4}+11553 t u^{5}+1257 u^{6}\right)\right]-m_{Z}^{8}\left[1864 s^{8}+10820 s^{7}(t+u)+4 s^{6}\left(6847 t^{2}\right.\right.$ $\left.+14418 t u+6847 u^{2}\right)+2 s^{5}(t+u)\left(17033 t^{2}+39274 t u+17033 u^{2}\right)+s^{4}\left(14141 t^{4}+71138 t^{3} u+111826 t^{2} u^{2}+71138 t u^{3}\right.$ $\left.+14141 u^{4}\right)-2 s^{3}(t+u)\left(5695 t^{4}+21723 t^{3} u+37444 t^{2} u^{2}+21723 t u^{3}+5695 u^{4}\right)-s^{2}\left(14753 t^{6}+104550 t^{5} u\right.$ $\left.+300227 t^{4} u^{2}+421692 t^{3} u^{3}+300227 t^{2} u^{4}+104550 t u^{5}+14753 u^{6}\right)-2 s(t+u)\left(2771 t^{6}+23944 t^{5} u+75106 t^{4} u^{2}\right.$ $\left.+108454 t^{3} u^{3}+75106 t^{2} u^{4}+23944 t u^{5}+2771 u^{6}\right)-2(t+u)^{2}\left(331 t^{6}+3842 t^{5} u+13494 t^{4} u^{2}+19926 t^{3} u^{3}+13494 t^{2} u^{4}\right.$ $\left.\left.+3842 t u^{5}+331 u^{6}\right)\right]+2 m_{Z}^{6}\left[168 s^{9}+1158 s^{8}(t+u)+12 s^{7}\left(316 t^{2}+671 t u+316 u^{2}\right)+4 s^{6}(t+u)\left(1693 t^{2}+3990 t u\right.\right.$ $\left.+1693 u^{2}\right)+s^{5}\left(5796 t^{4}+29542 t^{3} u+47188 t^{2} u^{2}+29542 t u^{3}+5796 u^{4}\right)+2 s^{4}(t+u)\left(162 t^{4}+4996 t^{3} u+8301 t^{2} u^{2}\right.$ $\left.+4996 t u^{3}+162 u^{4}\right)-s^{3}\left(3347 t^{6}+16010 t^{5} u+39319 t^{4} u^{2}+53548 t^{3} u^{3}+39319 t^{2} u^{4}+16010 t u^{5}+3347 u^{6}\right)-s^{2}(t+u)$ $\times\left(2439 t^{6}+16826 t^{5} u+49619 t^{4} u^{2}+71420 t^{3} u^{3}+49619 t^{2} u^{4}+16826 t u^{5}+2439 u^{6}\right)-s\left(645 t^{8}+7666 t^{7} u+35040 t^{6} u^{2}\right.$ $\left.+82140 t^{5} u^{3}+108250 t^{4} u^{4}+82140 t^{3} u^{5}+35040 t^{2} u^{6}+7666 t u^{7}+645 u^{8}\right)-(t+u)^{3}\left(51 t^{6}+852 t^{5} u+3539 t^{4} u^{2}\right.$ 
$\left.\left.+5396 t^{3} u^{3}+3539 t^{2} u^{4}+852 t u^{5}+51 u^{6}\right)\right]-m_{Z}^{4}\left[24 s^{10}+212 s^{9}(t+u)+4 s^{8}\left(241 t^{2}+512 t u+241 u^{2}\right)+2 s^{7}(t+u)\left(1179 t^{2}\right.\right.$

$\left.+2822 t u+1179 u^{2}\right)+s^{6}\left(2855 t^{4}+15364 t^{3} u+24930 t^{2} u^{2}+15364 t u^{3}+2855 u^{4}\right)+2 s^{5}(t+u)\left(367 t^{4}+6085 t^{3} u\right.$

$\left.+10618 t^{2} u^{2}+6085 t u^{3}+367 u^{4}\right)-s^{4}\left(2117 t^{6}+1876 t^{5} u-6829 t^{4} u^{2}-13152 t^{3} u^{3}-6829 t^{2} u^{4}+1876 t u^{5}+2117 u^{6}\right)$

$-2 s^{3}(t+u)\left(1241 t^{6}+4958 t^{5} u+11056 t^{4} u^{2}+15114 t^{3} u^{3}+11056 t^{2} u^{4}+4958 t u^{5}+1241 u^{6}\right)-s^{2}\left(1079 t^{8}+9328 t^{7} u\right.$

$\left.+36475 t^{6} u^{2}+80988 t^{5} u^{3}+105492 t^{4} u^{4}+80988 t^{3} u^{5}+36475 t^{2} u^{6}+9328 t u^{7}+1079 u^{8}\right)-2 s(t+u)\left(91 t^{8}+1293 t^{7} u\right.$

$\left.+6382 t^{6} u^{2}+15744 t^{5} u^{3}+21112 t^{4} u^{4}+15744 t^{3} u^{5}+6382 t^{2} u^{6}+1293 t u^{7}+91 u^{8}\right)-(t+u)^{4}\left(7 t^{6}+228 t^{5} u+1252 t^{4} u^{2}\right.$

$\left.\left.+1952 t^{3} u^{3}+1252 t^{2} u^{4}+228 t u^{5}+7 u^{6}\right)\right]+2 m_{Z}^{2}\left[16 s^{9}(t+u)^{2}+2 s^{8}(t+u)\left(35 t^{2}+82 t u+35 u^{2}\right)+s^{7}\left(95 t^{4}+627 t^{3} u\right.\right.$

$\left.+1056 t^{2} u^{2}+627 t u^{3}+95 u^{4}\right)-s^{6}(t+u)\left(57 t^{4}-721 t^{3} u-1422 t^{2} u^{2}-721 t u^{3}+57 u^{4}\right)-s^{5}\left(332 t^{6}+227 t^{5} u-1538 t^{4} u^{2}\right.$

$\left.-2874 t^{3} u^{3}-1538 t^{2} u^{4}+227 t u^{5}+332 u^{6}\right)-s^{4}(t+u)\left(424 t^{6}+911 t^{5} u+608 t^{4} u^{2}+298 t^{3} u^{3}+608 t^{2} u^{4}+911 t u^{5}+424 u^{6}\right)$

$-s^{3}\left(253 t^{8}+1415 t^{7} u+3802 t^{6} u^{2}+6836 t^{5} u^{3}+8376 t^{4} u^{4}+6836 t^{3} u^{5}+3802 t^{2} u^{6}+1415 t u^{7}+253 u^{8}\right)-s^{2}(t+u)\left(69 t^{8}\right.$

$\left.+611 t^{7} u+2282 t^{6} u^{2}+5134 t^{5} u^{3}+6750 t^{4} u^{4}+5134 t^{3} u^{5}+2282 t^{2} u^{6}+611 t u^{7}+69 u^{8}\right)-s(t+u)^{2}\left(6 t^{8}+129 t^{7} u\right.$

$\left.+682 t^{6} u^{2}+1796 t^{5} u^{3}+2452 t^{4} u^{4}+1796 t^{3} u^{5}+682 t^{2} u^{6}+129 t u^{7}+6 u^{8}\right)-t u(t+u)^{5}\left(7 t^{4}+68 t^{3} u+105 t^{2} u^{2}+68 t u^{3}\right.$

$\left.\left.+7 u^{4}\right)\right]+(t+u)(s+t+u)^{2}\left[s^{6}(t+u)\left(7 t^{2}-4 t u+7 u^{2}\right)+2 s^{5}\left(14 t^{4}+9 t^{3} u-4 t^{2} u^{2}+9 t u^{3}+14 u^{4}\right)+2 s^{4}(t+u)\left(21 t^{4}\right.\right.$

$\left.+3 t^{3} u-4 t^{2} u^{2}+3 t u^{3}+21 u^{4}\right)+2 s^{3}(t+u)^{2}\left(14 t^{4}+5 t^{3} u+10 t^{2} u^{2}+5 t u^{3}+14 u^{4}\right)+s^{2}(t+u)\left(7 t^{6}+38 t^{5} u+64 t^{4} u^{2}\right.$

$\left.+116 t^{3} u^{3}+64 t^{2} u^{4}+38 t u^{5}+7 u^{6}\right)+2 s t u\left(6 t^{6}+25 t^{5} u+69 t^{4} u^{2}+94 t^{3} u^{3}+69 t^{2} u^{4}+25 t u^{5}+6 u^{6}\right)+t^{2} u^{2}(t+u)^{3}\left(7 t^{2}\right.$

$\left.\left.+10 t u+7 u^{2}\right)\right]$,

$$
\begin{gathered}
\frac{d \sigma}{d t}\left(\gamma g \rightarrow Q \bar{Q}\left[\varsigma^{(8)}\right] Z\right)=\frac{9}{32} \frac{\alpha_{s}}{\alpha} \frac{d \sigma}{d t}\left(\gamma \gamma \rightarrow Q \bar{Q}\left[\varsigma^{(1)}\right] Z\right), \quad \varsigma={ }^{1} S_{0},{ }^{3} S_{1},{ }^{1} P_{1}, \\
\frac{d \sigma}{d t}\left(\gamma g \rightarrow Q \bar{Q}\left[{ }^{3} P_{J}^{(8)}\right] Z\right)=\frac{9}{32} \frac{\alpha_{s}}{\alpha} \sum_{J^{\prime}=0}^{2}\left(2 J^{\prime}+1\right) \frac{d \sigma}{d t}\left(\gamma \gamma \rightarrow Q \bar{Q}\left[{ }^{3} P_{J^{\prime}}^{(1)}\right] Z\right), \\
\frac{d \sigma}{d t}(g g \rightarrow Q \bar{Q}[n] Z)=\frac{9 \alpha_{s}^{2}}{512 \alpha^{2}} \frac{d \sigma}{d t}(\gamma \gamma \rightarrow Q \bar{Q}[n] Z), \quad n={ }^{1} S_{0}^{(1)},{ }^{3} S_{1}^{(1)},{ }^{1} P_{1}^{(1)},{ }^{3} P_{J}^{(1)},
\end{gathered}
$$

$$
\begin{aligned}
\frac{d \sigma}{d t}(g g \rightarrow & \left.Q \bar{Q}\left[{ }^{1} S_{0}^{(8)}\right] Z\right)=\frac{\pi \alpha_{s}^{2} g^{2}}{12 M m_{Z}^{2} s^{3}\left(m_{Z}^{2}-s-t\right)^{2}\left(m_{Z}^{2}-s-u\right)^{2}\left(2 m_{Z}^{2}-t-u\right)^{2}}\left(9 v _ { Q ^ { 2 } } ^ { 2 } m _ { Z } ^ { 2 } \left\{9 m_{Z}^{12}-3 m_{Z}^{10}[8 s+9(t+u)]+m_{Z}^{8}\left[24 s^{2}\right.\right.\right. \\
& \left.+56 s(t+u)+3\left(11 t^{2}+23 t u+11 u^{2}\right)\right]-m_{Z}^{6}\left[10 s^{3}+42 s^{2}(t+u)+2 s\left(25 t^{2}+54 t u+25 u^{2}\right)+3(t+u)\left(7 t^{2}+16 t u\right.\right. \\
& \left.\left.+7 u^{2}\right)\right]+m_{Z}^{4}\left[s^{4}+12 s^{3}(t+u)+s^{2}\left(25 t^{2}+58 t u+25 u^{2}\right)+4 s(t+u)\left(5 t^{2}+14 t u+5 u^{2}\right)+7 t^{4}+35 t^{3} u+51 t^{2} u^{2}+35 t u^{3}\right. \\
& \left.+7 u^{4}\right]-m_{Z}^{2}\left[s^{4}(t+u)+2 s^{3}\left(2 t^{2}+5 t u+2 u^{2}\right)+5 s^{2}(t+u)\left(t^{2}+4 t u+u^{2}\right)+s(t+u)^{2}\left(3 t^{2}+16 t u+3 u^{2}\right)+(t+u)\right. \\
& \left.\left.\times\left(t^{2}+t u+u^{2}\right)\left(t^{2}+7 t u+u^{2}\right)\right]+t u\left[s^{4}+2 s^{3}(t+u)+3 s^{2}(t+u)^{2}+2 s(t+u)^{3}+\left(t^{2}+t u+u^{2}\right)^{2}\right]\right\}+5 a_{Q}^{2} s \\
& \left.\times\left(2 m_{Z}^{2}-s-t-u\right)^{2}\left[m_{Z}^{8}-2 m_{Z}^{6}(t+u)-m_{Z}^{4}\left(s^{2}-t^{2}-4 t u-u^{2}\right)-2 m_{Z}^{2} t u(t+u)+t^{2} u^{2}\right]\right),
\end{aligned}
$$

$$
\begin{aligned}
\frac{d \sigma}{d t}(g g \rightarrow & \left.Q \bar{Q}\left[{ }^{3} S_{1}^{(8)}\right] Z\right)=\frac{-\pi \alpha_{s}^{2} g^{2}}{36 M m_{Z}^{2} s^{3}\left(m_{Z}^{2}-s-t\right)^{2}\left(m_{Z}^{2}-s-u\right)^{2}\left(2 m_{Z}^{2}-t-u\right)^{2}}\left(5 v _ { Q } ^ { 2 } m _ { Z } ^ { 2 } s \left\{m_{Z}^{10}-4 m_{Z}^{8}(3 s+t+u)+m_{Z}^{6}\left[22 s^{2}\right.\right.\right. \\
& \left.+26 s(t+u)+5 t^{2}+12 t u+5 u^{2}\right]-2 m_{Z}^{4}\left[5 s^{3}+14 s^{2}(t+u)+s\left(8 t^{2}+23 t u+8 u^{2}\right)+(t+u)\left(t^{2}+5 t u+u^{2}\right)\right] \\
& -m_{Z}^{2}\left[s^{4}-4 s^{3}(t+u)-s^{2}\left(9 t^{2}+26 t u+9 u^{2}\right)-2 s(t+u)\left(t^{2}+10 t u+u^{2}\right)-t u\left(4 t^{2}+9 t u+4 u^{2}\right)\right]-2\left[s^{3}\left(t^{2}+t u+u^{2}\right)\right.
\end{aligned}
$$


$\left.\left.+s^{2}(t+u)^{3}+s t u\left(t^{2}+3 t u+u^{2}\right)+t^{2} u^{2}(t+u)\right]\right\}-9 a_{Q}^{2}\left\{9 m_{Z}^{12}(s+t+u)-m_{Z}^{10}\left[18 s^{2}+49 s(t+u)+27(t+u)^{2}\right]\right.$

$+m_{Z}^{8}\left[7 s^{3}+57 s^{2}(t+u)+s\left(83 t^{2}+169 t u+83 u^{2}\right)+3(t+u)\left(11 t^{2}+23 t u+11 u^{2}\right)\right]+m_{Z}^{6}\left[2 s^{4}-13 s^{3}(t+u)-2 s^{2}\left(31 t^{2}\right.\right.$

$\left.\left.+63 t u+31 u^{2}\right)-5 s(t+u)\left(13 t^{2}+28 t u+13 u^{2}\right)-3(t+u)^{2}\left(7 t^{2}+16 t u+7 u^{2}\right)\right]-m_{Z}^{4}\left[2 s^{4}(t+u)-s^{3}\left(8 t^{2}+19 t u\right.\right.$

$\left.+8 u^{2}\right)-5 s^{2}(t+u)(3 t+2 u)(2 t+3 u)-5 s\left(5 t^{4}+23 t^{3} u+35 t^{2} u^{2}+23 t u^{3}+5 u^{4}\right)-(t+u)\left(7 t^{4}+35 t^{3} u+51 t^{2} u^{2}\right.$

$\left.\left.+35 t u^{3}+7 u^{4}\right)\right]+m_{Z}^{2}\left[s^{4}\left(t^{2}+u^{2}\right)-2 s^{3}(t+u)\left(t^{2}+3 t u+u^{2}\right)-s^{2}(t+u)^{2}\left(6 t^{2}+17 t u+6 u^{2}\right)-s(t+u)\left(4 t^{4}+26 t^{3} u\right.\right.$

$\left.\left.\left.\left.+37 t^{2} u^{2}+26 t u^{3}+4 u^{4}\right)-(t+u)^{2}\left(t^{4}+8 t^{3} u+9 t^{2} u^{2}+8 t u^{3}+u^{4}\right)\right]+t u(s+t+u)\left[s(t+u)+t^{2}+t u+u^{2}\right]^{2}\right\}\right)$,

$$
\begin{aligned}
& \frac{d \sigma}{d t}\left(g g \rightarrow Q \bar{Q}\left[{ }^{1} P_{1}^{(8)}\right] Z\right)=\frac{-\pi \alpha_{s}^{2} g^{2}}{9 M^{3} m_{Z}^{2} s^{3}\left(m_{Z}^{2}-s-t\right)^{3}\left(m_{Z}^{2}-s-u\right)^{3}\left(2 m_{Z}^{2}-t-u\right)^{4}}\left(1 0 v _ { Q } ^ { 2 } m _ { Z } ^ { 2 } s \left\{48 m_{Z}^{18}-16 m_{Z}^{16}[11 s+15(t+u)]\right.\right. \\
& +2 m_{Z}^{14}\left[141 s^{2}+374 s(t+u)+4\left(65 t^{2}+134 t u+65 u^{2}\right)\right]-4 m_{Z}^{12}\left[65 s^{3}+255 s^{2}(t+u)+s\left(341 t^{2}\right.\right. \\
& \left.\left.+696 t u+341 u^{2}\right)+32(t+u)\left(5 t^{2}+11 t u+5 u^{2}\right)\right]+m_{Z}^{10}\left[138 s^{4}+798 s^{3}(t+u)+10 s^{2}\left(158 t^{2}\right.\right. \\
& \left.+315 t u+158 u^{2}\right)+s(t+u)\left(1399 t^{2}+2958 t u+1399 u^{2}\right)+491 t^{4}+2188 t^{3} u+3378 t^{2} u^{2}+2188 t u^{3} \\
& \left.+491 u^{4}\right]-m_{Z}^{8}\left[24 s^{5}+356 s^{4}(t+u)+20 s^{3}\left(53 t^{2}+101 t u+53 u^{2}\right)+10 s^{2}(t+u)\left(138 t^{2}+265 t u\right.\right. \\
& \left.+138 u^{2}\right)+s(t+u)^{2}\left(887 t^{2}+1956 t u+887 u^{2}\right)+(t+u)\left(241 t^{4}+1188 t^{3} u+1846 t^{2} u^{2}+1188 t u^{3}\right. \\
& \left.\left.+241 u^{4}\right)\right]-m_{Z}^{6}\left[18 s^{6}-60 s^{5}(t+u)-s^{4}\left(417 t^{2}+734 t u+417 u^{2}\right)-s^{3}(t+u)\left(801 t^{2}+1298 t u\right.\right. \\
& \left.+801 u^{2}\right)-s^{2}\left(749 t^{4}+2772 t^{3} u+4068 t^{2} u^{2}+2772 t u^{3}+749 u^{4}\right)-s(t+u)\left(359 t^{4}+1534 t^{3} u\right. \\
& \left.\left.+2362 t^{2} u^{2}+1534 t u^{3}+359 u^{4}\right)-2(t+u)^{2}\left(37 t^{4}+217 t^{3} u+332 t^{2} u^{2}+217 t u^{3}+37 u^{4}\right)\right] \\
& +m_{Z}^{4}\left[12 s^{7}+16 s^{6}(t+u)-s^{5}\left(83 t^{2}+122 t u+83 u^{2}\right)-s^{4}(t+u)\left(275 t^{2}+356 t u+275 u^{2}\right)\right. \\
& -s^{3}\left(367 t^{4}+1153 t^{3} u+1580 t^{2} u^{2}+1153 t u^{3}+367 u^{4}\right)-s^{2}(t+u)\left(258 t^{4}+843 t^{3} u+1214 t^{2} u^{2}\right. \\
& \left.+843 t u^{3}+258 u^{4}\right)-4 s\left(23 t^{6}+143 t^{5} u+371 t^{4} u^{2}+500 t^{3} u^{3}+371 t^{2} u^{4}+143 t u^{5}+23 u^{6}\right) \\
& \left.-(t+u)^{3}\left(13 t^{4}+104 t^{3} u+150 t^{2} u^{2}+104 t u^{3}+13 u^{4}\right)\right]-m_{Z}^{2}\left[2 s^{8}+6 s^{7}(t+u)-s^{6}\left(7 t^{2}+4 t u\right.\right. \\
& \left.+7 u^{2}\right)-52 s^{5}(t+u)\left(t^{2}+t u+u^{2}\right)-s^{4}\left(97 t^{4}+269 t^{3} u+346 t^{2} u^{2}+269 t u^{3}+97 u^{4}\right)-2 s^{3}(t+u) \\
& \times\left(48 t^{4}+122 t^{3} u+155 t^{2} u^{2}+122 t u^{3}+48 u^{4}\right)-s^{2}\left(53 t^{6}+244 t^{5} u+531 t^{4} u^{2}+674 t^{3} u^{3}+531 t^{2} u^{4}\right. \\
& \left.+244 t u^{5}+53 u^{6}\right)-s(t+u)\left(14 t^{6}+82 t^{5} u+217 t^{4} u^{2}+290 t^{3} u^{3}+217 t^{2} u^{4}+82 t u^{5}+14 u^{6}\right) \\
& \left.-(t+u)^{4}\left(t^{4}+15 t^{3} u+19 t^{2} u^{2}+15 t u^{3}+u^{4}\right)\right]-(t+u)\left[s^{7}(t+u)+s^{6}\left(5 t^{2}+6 t u+5 u^{2}\right)\right. \\
& +s^{5}(t+u)\left(11 t^{2}+8 t u+11 u^{2}\right)+2 s^{4}\left(7 t^{4}+16 t^{3} u+19 t^{2} u^{2}+16 t u^{3}+7 u^{4}\right)+s^{3}(t+u) \\
& \times\left(11 t^{4}+21 t^{3} u+25 t^{2} u^{2}+21 t u^{3}+11 u^{4}\right)+s^{2}\left(5 t^{6}+19 t^{5} u+38 t^{4} u^{2}+46 t^{3} u^{3}+38 t^{2} u^{4}+19 t u^{5}\right. \\
& \left.\left.\left.+5 u^{6}\right)+s(t+u)\left(t^{6}+5 t^{5} u+14 t^{4} u^{2}+18 t^{3} u^{3}+14 t^{2} u^{4}+5 t u^{5}+u^{6}\right)+t u(t+u)^{4}\left(t^{2}+t u+u^{2}\right)\right]\right\} \\
& +9 a_{Q}^{2}\left\{36 m_{Z}^{20}(s-t-u)-4 m_{Z}^{18}\left[17 s^{2}+8 s(t+u)-45(t+u)^{2}\right]+m_{Z}^{16}\left[28 s^{3}+12 s^{2}(t+u)\right.\right. \\
& \left.-s\left(257 t^{2}+466 t u+257 u^{2}\right)-3(t+u)\left(131 t^{2}+278 t u+131 u^{2}\right)\right]-4 m_{Z}^{14}\left[2 s^{4}-40 s^{3}(t+u)\right. \\
& \left.-6 s^{2}\left(22 t^{2}+41 t u+22 u^{2}\right)-4 s(t+u)\left(47 t^{2}+88 t u+47 u^{2}\right)-3(t+u)^{2}\left(41 t^{2}+98 t u+41 u^{2}\right)\right] \\
& +m_{Z}^{12}\left[28 s^{5}-88 s^{4}(t+u)-s^{3}\left(695 t^{2}+1334 t u+695 u^{2}\right)-s^{2}(t+u)\left(1201 t^{2}+2218 t u+1201 u^{2}\right)\right. \\
& -2 s\left(485 t^{4}+1938 t^{3} u+2910 t^{2} u^{2}+1938 t u^{3}+485 u^{4}\right)-4(t+u)\left(97 t^{4}+473 t^{3} u+750 t^{2} u^{2}\right. \\
& \left.\left.+473 t u^{3}+97 u^{4}\right)\right]-m_{Z}^{10}\left[20 s^{6}+44 s^{5}(t+u)-26 s^{4}\left(11 t^{2}+20 t u+11 u^{2}\right)-8 s^{3}(t+u)\left(132 t^{2}\right.\right. \\
& \left.+241 t u+132 u^{2}\right)-s^{2}\left(1277 t^{4}+4942 t^{3} u+7386 t^{2} u^{2}+4942 t u^{3}+1277 u^{4}\right)-2 s(t+u)\left(363 t^{4}\right. \\
& \left.+1544 t^{3} u+2346 t^{2} u^{2}+1544 t u^{3}+363 u^{4}\right)-6(t+u)^{2}\left(33 t^{4}+190 t^{3} u+310 t^{2} u^{2}+190 t u^{3}\right. \\
& \left.\left.+33 u^{4}\right)\right]+m_{Z}^{8}\left[4 s^{7}+32 s^{6}(t+u)+s^{5}\left(7 t^{2}+30 t u+7 u^{2}\right)-s^{4}(t+u)\left(351 t^{2}+566 t u+351 u^{2}\right)\right.
\end{aligned}
$$




$$
\begin{aligned}
& -2 s^{3}\left(421 t^{4}+1577 t^{3} u+2354 t^{2} u^{2}+1577 t u^{3}+421 u^{4}\right)-s^{2}(t+u)\left(779 t^{4}+3112 t^{3} u+4734 t^{2} u^{2}\right. \\
& \left.+3112 t u^{3}+779 u^{4}\right)-2 s\left(168 t^{6}+1138 t^{5} u+2987 t^{4} u^{2}+4042 t^{3} u^{3}+2987 t^{2} u^{4}+1138 t u^{5}+168 u^{6}\right) \\
& \left.-2(t+u)\left(32 t^{6}+303 t^{5} u+915 t^{4} u^{2}+1280 t^{3} u^{3}+915 t^{2} u^{4}+303 t u^{5}+32 u^{6}\right)\right]-m_{Z}^{6}\left[4 s^{7}(t+u)\right. \\
& +2 s^{6}\left(9 t^{2}+16 t u+9 u^{2}\right)-4 s^{5}(t+u)\left(5 t^{2}+6 t u+5 u^{2}\right)-2 s^{4}\left(107 t^{4}+374 t^{3} u+550 t^{2} u^{2}+374 t u^{3}\right. \\
& \left.+107 u^{4}\right)-3 s^{3}(t+u)\left(127 t^{4}+478 t^{3} u+742 t^{2} u^{2}+478 t u^{3}+127 u^{4}\right)-2 s^{2}\left(141 t^{6}+897 t^{5} u\right. \\
& \left.+2309 t^{4} u^{2}+3134 t^{3} u^{3}+2309 t^{2} u^{4}+897 t u^{5}+141 u^{6}\right)-s(t+u)\left(95 t^{6}+746 t^{5} u+2017 t^{4} u^{2}\right. \\
& \left.+2764 t^{3} u^{3}+2017 t^{2} u^{4}+746 t u^{5}+95 u^{6}\right)-4(t+u)^{2}\left(3 t^{6}+40 t^{5} u+134 t^{4} u^{2}+186 t^{3} u^{3}+134 t^{2} u^{4}\right. \\
& \left.\left.+40 t u^{5}+3 u^{6}\right)\right]+m_{Z}^{4}\left[s^{7}(t+u)^{2}+s^{6}(t+u)\left(5 t^{2}+2 t u+5 u^{2}\right)-2 s^{5}\left(5 t^{4}+24 t^{3} u+36 t^{2} u^{2}\right.\right. \\
& \left.+24 t u^{3}+5 u^{4}\right)-s^{4}(t+u)\left(65 t^{4}+240 t^{3} u+374 t^{2} u^{2}+240 t u^{3}+65 u^{4}\right)-s^{3}\left(94 t^{6}+577 t^{5} u\right. \\
& \left.+1489 t^{4} u^{2}+2060 t^{3} u^{3}+1489 t^{2} u^{4}+577 t u^{5}+94 u^{6}\right)-s^{2}(t+u)\left(57 t^{6}+424 t^{5} u+1100 t^{4} u^{2}\right. \\
& \left.+1578 t^{3} u^{3}+1100 t^{2} u^{4}+424 t u^{5}+57 u^{6}\right)-s\left(15 t^{8}+186 t^{7} u+758 t^{6} u^{2}+1626 t^{5} u^{3}+2074 t^{4} u^{4}\right. \\
& \left.+1626 t^{3} u^{5}+758 t^{2} u^{6}+186 t u^{7}+15 u^{8}\right)-(t+u)\left(t^{8}+28 t^{7} u+160 t^{6} u^{2}+376 t^{5} u^{3}+490 t^{4} u^{4}\right. \\
& \left.\left.+376 t^{3} u^{5}+160 t^{2} u^{6}+28 t u^{7}+u^{8}\right)\right]-m_{Z}^{2}\left[s^{6}(t-u)^{2}(t+u)^{2}-s^{5}(t+u)\left(t^{4}+12 t^{3} u+18 t^{2} u^{2}\right.\right. \\
& \left.+12 t u^{3}+u^{4}\right)-2 s^{4}\left(4 t^{6}+29 t^{5} u+77 t^{4} u^{2}+110 t^{3} u^{3}+77 t^{2} u^{4}+29 t u^{5}+4 u^{6}\right)-2 s^{3}(t+u) \\
& \times\left(5 t^{6}+42 t^{5} u+106 t^{4} u^{2}+170 t^{3} u^{3}+106 t^{2} u^{4}+42 t u^{5}+5 u^{6}\right)-s^{2}\left(5 t^{8}+69 t^{7} u+261 t^{6} u^{2}\right. \\
& \left.+577 t^{5} u^{3}+756 t^{4} u^{4}+577 t^{3} u^{5}+261 t^{2} u^{6}+69 t u^{7}+5 u^{8}\right)-s(t+u)^{3}\left(t^{6}+19 t^{5} u+53 t^{4} u^{2}\right. \\
& \left.+78 t^{3} u^{3}+53 t^{2} u^{4}+19 t u^{5}+u^{6}\right)-2 t u(t+u)^{2}\left(t^{6}+9 t^{5} u+21 t^{4} u^{2}+28 t^{3} u^{3}+21 t^{2} u^{4}+9 t u^{5}\right. \\
& \left.\left.+u^{6}\right)\right]-t u(t+u)\left[s^{5}(t+u)\left(t^{2}+t u+u^{2}\right)+s^{4}\left(4 t^{4}+9 t^{3} u+18 t^{2} u^{2}+9 t u^{3}+4 u^{4}\right)+2 s^{3}(t+u)\right. \\
& \times\left(3 t^{4}+5 t^{3} u+14 t^{2} u^{2}+5 t u^{3}+3 u^{4}\right)+2 s^{2}(t+u)^{2}\left(2 t^{4}+3 t^{3} u+9 t^{2} u^{2}+3 t u^{3}+2 u^{4}\right) \\
& \left.\left.\left.+s(t+u)\left(t^{3}+3 t^{2} u+2 t u^{2}+u^{3}\right)\left(t^{3}+2 t^{2} u+3 t u^{2}+u^{3}\right)+t u(t+u)^{2}\left(t^{2}+t u+u^{2}\right)^{2}\right]\right\}\right),
\end{aligned}
$$

$$
\begin{aligned}
\frac{d \sigma}{d t}\left(g g \rightarrow Q \bar{Q}\left[{ }^{3} P_{J}^{(8)}\right] Z\right)= & \frac{\pi \alpha_{s}^{2} g^{2}}{3 M^{3} m_{Z}^{2} s^{3}\left(m_{Z}^{2}-s-t\right)^{3}\left(m_{Z}^{2}-s-u\right)^{3}\left(2 m_{Z}^{2}-t-u\right)^{4}}\left(9 v _ { Q } ^ { 2 } m _ { Z } ^ { 2 } \left\{72 m_{Z}^{20}-12 m_{Z}^{18}[31 s+33(t+u)]\right.\right. \\
& +m_{Z}^{16}\left[760 s^{2}+1788 s(t+u)+975 t^{2}+2046 t u+975 u^{2}\right]-m_{Z}^{14}\left[804 s^{3}+3144 s^{2}(t+u)+s\left(3785 t^{2}\right.\right. \\
& \left.\left.+7854 t u+3785 u^{2}\right)+108(t+u)\left(13 t^{2}+30 t u+13 u^{2}\right)\right]+m_{Z}^{12}\left[520 s^{4}+2868 s^{3}(t+u)+s^{2}\left(5661 t^{2}\right.\right. \\
& \left.+11578 t u+5661 u^{2}\right)+4 s(t+u)\left(1151 t^{2}+2569 t u+1151 u^{2}\right)+4\left(323 t^{4}+1513 t^{3} u+2376 t^{2} u^{2}\right. \\
& \left.\left.+1513 t u^{3}+323 u^{4}\right)\right]-m_{Z}^{10}\left[236 s^{5}+1644 s^{4}(t+u)+s^{3}\left(4431 t^{2}+8902 t u+4431 u^{2}\right)+2 s^{2}(t+u)\right. \\
& \times\left(2885 t^{2}+6188 t u+2885 u^{2}\right)+s\left(3514 t^{4}+15863 t^{3} u+24534 t^{2} u^{2}+15863 t u^{3}+3514 u^{4}\right)+2(t+u) \\
& \left.\times\left(389 t^{4}+2086 t^{3} u+3366 t^{2} u^{2}+2086 t u^{3}+389 u^{4}\right)\right]+m_{Z}^{8}\left[56 s^{6}+628 s^{5}(t+u)+s^{4}\left(2199 t^{2}+4382 t u\right.\right. \\
& \left.+2199 u^{2}\right)+20 s^{3}(t+u)\left(190 t^{2}+393 t u+190 u^{2}\right)+s^{2}\left(3606 t^{4}+15715 t^{3} u+23922 t^{2} u^{2}+15715 t u^{3}\right. \\
& \left.+3606 u^{4}\right)+s(t+u)\left(1720 t^{4}+8657 t^{3} u+13402 t^{2} u^{2}+8657 t u^{3}+1720 u^{4}\right)+2\left(150 t^{6}+1301 t^{5} u\right. \\
& \left.\left.+3823 t^{4} u^{2}+5328 t^{3} u^{3}+3823 t^{2} u^{4}+1301 t u^{5}+150 u^{6}\right)\right]+m_{Z}^{6}\left[4 s^{7}-96 s^{6}(t+u)-3 s^{5}\left(219 t^{2}\right.\right. \\
& \left.+442 t u+219 u^{2}\right)-4 s^{4}(t+u)\left(387 t^{2}+800 t u+387 u^{2}\right)-4 s^{3}\left(479 t^{4}+2045 t^{3} u+3095 t^{2} u^{2}+2045 t u^{3}\right. \\
& \left.+479 u^{4}\right)-2 s^{2}(t+u)\left(695 t^{4}+3346 t^{3} u+5022 t^{2} u^{2}+3346 t u^{3}+695 u^{4}\right)-s\left(526 t^{6}+4237 t^{5} u\right. \\
& \left.+11717 t^{4} u^{2}+15936 t^{3} u^{3}+11717 t^{2} u^{4}+4237 t u^{5}+526 u^{6}\right)-4(t+u)\left(17 t^{6}+198 t^{5} u+636 t^{4} u^{2}\right. \\
& \left.\left.+890 t^{3} u^{3}+636 t^{2} u^{4}+198 t u^{5}+17 u^{6}\right)\right]-m_{Z}^{4}\left[4 s^{7}(t+u)-69 s^{6}(t+u)^{2}-20 s^{5}(t+u)\left(17 t^{2}+36 t u\right.\right. \\
& \left.+17 u^{2}\right)-2 s^{4}\left(297 t^{4}+1276 t^{3} u+1954 t^{2} u^{2}+1276 t u^{3}+297 u^{4}\right)-2 s^{3}(t+u)\left(276 t^{4}+1311 t^{3} u\right.
\end{aligned}
$$




$$
\begin{aligned}
& \left.+1996 t^{2} u^{2}+1311 t u^{3}+276 u^{4}\right)-s^{2}\left(312 t^{6}+2433 t^{5} u+6563 t^{4} u^{2}+8836 t^{3} u^{3}+6563 t^{2} u^{4}\right. \\
& \left.+2433 t u^{5}+312 u^{6}\right)-s(t+u)\left(92 t^{6}+947 t^{5} u+2734 t^{4} u^{2}+3626 t^{3} u^{3}+2734 t^{2} u^{4}+947 t u^{5}+92 u^{6}\right) \\
& \left.-7 t^{8}-164 t^{7} u-876 t^{6} u^{2}-2048 t^{5} u^{3}-2666 t^{4} u^{4}-2048 t^{3} u^{5}-876 t^{2} u^{6}-164 t u^{7}-7 u^{8}\right] \\
& +m_{Z}^{2}\left[s^{7}(t+u)^{2}-2 s^{6}(t+u)\left(11 t^{2}+28 t u+11 u^{2}\right)-s^{5}\left(80 t^{4}+387 t^{3} u+590 t^{2} u^{2}+387 t u^{3}+80 u^{4}\right)\right. \\
& -4 s^{4}(t+u)\left(26 t^{4}+143 t^{3} u+215 t^{2} u^{2}+143 t u^{3}+26 u^{4}\right)-s^{3}\left(72 t^{6}+635 t^{5} u+1741 t^{4} u^{2}+2396 t^{3} u^{3}\right. \\
& \left.+1741 t^{2} u^{4}+635 t u^{5}+72 u^{6}\right)-2 s^{2}(t+u)\left(16 t^{6}+180 t^{5} u+495 t^{4} u^{2}+672 t^{3} u^{3}+495 t^{2} u^{4}\right. \\
& \left.+180 t u^{5}+16 u^{6}\right)-s(t+u)^{2}\left(7 t^{6}+123 t^{5} u+376 t^{4} u^{2}+465 t^{3} u^{3}+376 t^{2} u^{4}+123 t u^{5}+7 u^{6}\right) \\
& \left.-2 t u(t+u)\left(t^{2}+t u+u^{2}\right)\left(7 t^{4}+48 t^{3} u+76 t^{2} u^{2}+48 t u^{3}+7 u^{4}\right)\right]+(t+u)\left[s ^ { 6 } ( t + u ) \left(2 t^{2}+11 t u\right.\right. \\
& \left.+2 u^{2}\right)+s^{5}\left(4 t^{4}+47 t^{3} u+70 t^{2} u^{2}+47 t u^{3}+4 u^{4}\right)+s^{4}(t+u)\left(2 t^{4}+61 t^{3} u+75 t^{2} u^{2}+61 t u^{3}\right. \\
& \left.+2 u^{4}\right)+s^{3} t u\left(47 t^{4}+132 t^{3} u+190 t^{2} u^{2}+132 t u^{3}+47 u^{4}\right)+s^{2} t u(t+u)\left(25 t^{4}+63 t^{3} u+93 t^{2} u^{2}\right. \\
& \left.+63 t u^{3}+25 u^{4}\right)+\operatorname{stu}\left(7 t^{6}+38 t^{5} u+78 t^{4} u^{2}+98 t^{3} u^{3}+78 t^{2} u^{4}+38 t u^{5}+7 u^{6}\right)+7 t^{2} u^{2}(t+u) \\
& \left.\left.\times\left(t^{2}+t u+u^{2}\right)^{2}\right]\right\}-10 a_{Q}^{2} s\left\{36 m_{Z}^{20}-8 m_{Z}^{18}[16 s+17(t+u)]+m_{Z}^{16}\left[212 s^{2}+320 s(t+u)+153 t^{2}\right.\right. \\
& \left.+382 t u+153 u^{2}\right]-m_{Z}^{14}\left[318 s^{3}+346 s^{2}(t+u)-2 s\left(11 t^{2}-114 t u+11 u^{2}\right)-(t+u)\left(67 t^{2}-150 t u\right.\right. \\
& \left.\left.+67 u^{2}\right)\right]+m_{Z}^{12}\left[440 s^{4}+636 s^{3}(t+u)-s^{2}\left(373 t^{2}+362 t u+373 u^{2}\right)-s(t+u)\left(915 t^{2}+1034 t u\right.\right. \\
& \left.\left.+915 u^{2}\right)-337 t^{4}-949 t^{3} u-1180 t^{2} u^{2}-949 t u^{3}-337 u^{4}\right]-m_{Z}^{10}\left[388 s^{5}+982 s^{4}(t+u)+2 s^{3}\right. \\
& \times\left(128 t^{2}+417 t u+128 u^{2}\right)-s^{2}(t+u)\left(1351 t^{2}+1864 t u+1351 u^{2}\right)-2 s\left(687 t^{4}+2335 t^{3} u+3216 t^{2} u^{2}\right. \\
& \left.\left.+2335 t u^{3}+687 u^{4}\right)-2(t+u)\left(187 t^{4}+636 t^{3} u+818 t^{2} u^{2}+636 t u^{3}+187 u^{4}\right)\right]+m_{Z}^{8}\left[188 s^{6}\right. \\
& +718 s^{5}(t+u)+2 s^{4}\left(421 t^{2}+946 t u+421 u^{2}\right)-2 s^{3}(t+u)\left(151 t^{2}+45 t u+151 u^{2}\right)-2 s^{2}\left(703 t^{4}\right. \\
& \left.+2496 t^{3} u+3508 t^{2} u^{2}+2496 t u^{3}+703 u^{4}\right)-s(t+u)\left(1003 t^{4}+3708 t^{3} u+5034 t^{2} u^{2}+3708 t u^{3}\right. \\
& \left.\left.+1003 u^{4}\right)-217 t^{6}-1323 t^{5} u-3116 t^{4} u^{2}-4016 t^{3} u^{3}-3116 t^{2} u^{4}-1323 t u^{5}-217 u^{6}\right] \\
& -m_{Z}^{6}\left[46 s^{7}+240 s^{6}(t+u)+5 s^{5}\left(101 t^{2}+220 t u+101 u^{2}\right)+s^{4}(t+u)\left(339 t^{2}+916 t u+339 u^{2}\right)\right. \\
& -2 s^{3}\left(196 t^{4}+627 t^{3} u+833 t^{2} u^{2}+627 t u^{3}+196 u^{4}\right)-s^{2}(t+u)\left(771 t^{4}+2854 t^{3} u+3968 t^{2} u^{2}\right. \\
& \left.+2854 t u^{3}+771 u^{4}\right)-s\left(421 t^{6}+2566 t^{5} u+6173 t^{4} u^{2}+8040 t^{3} u^{3}+6173 t^{2} u^{4}+2566 t u^{5}+421 u^{6}\right) \\
& \left.-(t+u)\left(72 t^{6}+524 t^{5} u+1295 t^{4} u^{2}+1674 t^{3} u^{3}+1295 t^{2} u^{4}+524 t u^{5}+72 u^{6}\right)\right]+m_{Z}^{4}\left[4 s^{8}+30 s^{7}\right. \\
& \times(t+u)+2 s^{6}\left(53 t^{2}+114 t u+53 u^{2}\right)+s^{5}(t+u)\left(163 t^{2}+388 t u+163 u^{2}\right)+s^{4}\left(29 t^{4}+237 t^{3} u\right. \\
& \left.+424 t^{2} u^{2}+237 t u^{3}+29 u^{4}\right)-2 s^{3}(t+u)^{3}\left(108 t^{2}+127 t u+108 u^{2}\right)-s^{2}\left(254 t^{6}+1423 t^{5} u\right. \\
& \left.+3385 t^{4} u^{2}+4416 t^{3} u^{3}+3385 t^{2} u^{4}+1423 t u^{5}+254 u^{6}\right)-s(t+u)\left(105 t^{6}+682 t^{5} u+1694 t^{4} u^{2}\right. \\
& \left.+2206 t^{3} u^{3}+1694 t^{2} u^{4}+682 t u^{5}+105 u^{6}\right)-(t+u)^{2}\left(13 t^{6}+126 t^{5} u+331 t^{4} u^{2}+427 t^{3} u^{3}\right. \\
& \left.\left.+331 t^{2} u^{4}+126 t u^{5}+13 u^{6}\right)\right]-m_{Z}^{2}\left[5 s^{7}(t+u)^{2}+s^{6}(t+u)\left(17 t^{2}+38 t u+17 u^{2}\right)+s^{5}\left(11 t^{4}+67 t^{3} u\right.\right. \\
& \left.+110 t^{2} u^{2}+67 t u^{3}+11 u^{4}\right)-s^{4}(t+u)\left(3 t^{2}+5 t u+3 u^{2}\right)\left(11 t^{2}+2 t u+11 u^{2}\right)-s^{3}\left(69 t^{6}+310 t^{5} u\right. \\
& \left.+677 t^{4} u^{2}+866 t^{3} u^{3}+677 t^{2} u^{4}+310 t u^{5}+69 u^{6}\right)-s^{2}(t+u)\left(51 t^{6}+252 t^{5} u+598 t^{4} u^{2}+776 t^{3} u^{3}\right. \\
& \left.+598 t^{2} u^{4}+252 t u^{5}+51 u^{6}\right)-s(t+u)^{2}\left(15 t^{6}+99 t^{5} u+259 t^{4} u^{2}+336 t^{3} u^{3}+259 t^{2} u^{4}+99 t u^{5}\right. \\
& \left.\left.+15 u^{6}\right)-(t+u)^{3}\left(t^{6}+17 t^{5} u+48 t^{4} u^{2}+62 t^{3} u^{3}+48 t^{2} u^{4}+17 t u^{5}+u^{6}\right)\right]-(t+u)(s+t+u)^{2} \\
& \times\left[s^{4}(t+u)\left(t^{2}+t u+u^{2}\right)+s^{3}\left(3 t^{4}+6 t^{3} u+8 t^{2} u^{2}+6 t u^{3}+3 u^{4}\right)+s^{2}(t+u)\left(3 t^{4}+4 t^{3} u+9 t^{2} u^{2}\right.\right. \\
& \left.\left.\left.\left.+4 t u^{3}+3 u^{4}\right)+s\left(t^{6}+4 t^{5} u+11 t^{4} u^{2}+14 t^{3} u^{3}+11 t^{2} u^{4}+4 t u^{5}+u^{6}\right)+t u(t+u)^{3}\left(t^{2}+t u+u^{2}\right)\right]\right\}\right),
\end{aligned}
$$

$$
\frac{d \sigma}{d t}\left(q \bar{q} \rightarrow Q \bar{Q}\left[{ }^{1} S_{0}^{(8)}\right] Z\right)=\frac{4 \pi \alpha_{s}^{2} g^{2} v_{Q}^{2}}{9 M s^{3}\left(2 m_{Z}^{2}-t-u\right)^{2}}\left[2 m_{Z}^{4}-2 m_{Z}^{2}(s+t+u)+t^{2}+u^{2}\right]
$$




$$
\begin{aligned}
& \frac{d \sigma}{d t}\left(q \bar{q} \rightarrow Q \bar{Q}\left[{ }^{3} S_{1}^{(8)}\right] Z\right)=\frac{-2 \pi \alpha_{s}^{2} g^{2}}{27 M^{5} m_{Z}^{2} s^{3} t^{2} u^{2}\left(2 m_{Z}^{2}-t-u\right)^{2}}\left(( v _ { q } ^ { 2 } + a _ { q } ^ { 2 } ) m _ { Z } ^ { 2 } s ( 2 m _ { Z } ^ { 2 } - t - u ) ^ { 2 } \left\{m_{Z}^{6}\left(t^{2}+u^{2}\right)-2 m_{Z}^{4}(s+t+u)\right.\right. \\
& \times\left(t^{2}+u^{2}\right)+m_{Z}^{2}\left[s^{2}(t+u)^{2}+2 s(t+u)\left(t^{2}+t u+u^{2}\right)+\left(t^{2}+u^{2}\right)\left(t^{2}+3 t u+u^{2}\right)\right]-t u\left[2 s^{3}+4 s^{2}(t+u)\right. \\
& \left.\left.+s\left(3 t^{2}+4 t u+3 u^{2}\right)+(t+u)\left(t^{2}+u^{2}\right)\right]\right\}-4 a_{q} a_{Q} M^{2} m_{Z}^{2} s t u\left(2 m_{Z}^{2}-t-u\right)(t+u)\left[2 m_{Z}^{4}-3 m_{Z}^{2}(s+t+u)\right. \\
& \left.\left.+(s+t+u)^{2}\right]+2 a_{Q}^{2} M^{4} t^{2} u^{2}\left\{2 m_{Z}^{4}(3 s-t-u)-m_{Z}^{2}\left[6 s^{2}+4 s(t+u)-2(t+u)^{2}\right]-(s+t+u)\left(t^{2}+u^{2}\right)\right\}\right), \\
& \frac{d \sigma}{d t}\left(q \bar{q} \rightarrow Q \bar{Q}\left[{ }^{1} P_{1}^{(8)}\right] Z\right)=\frac{-16 \pi \alpha_{s}^{2} g^{2} a_{Q}^{2}}{27 M^{3} m_{Z}^{2} s^{3}\left(2 m_{Z}^{2}-t-u\right)^{4}}\left\{8 m_{Z}^{8}(s-t-u)-16 m_{Z}^{6}\left[s^{2}-(t+u)^{2}\right]+2 m_{Z}^{4}\left[4 s^{3}+4 s^{2}(t+u)\right.\right. \\
& \left.-s\left(3 t^{2}+10 t u+3 u^{2}\right)-(t+u)\left(7 t^{2}+10 t u+7 u^{2}\right)\right]-2 m_{Z}^{2}\left[s^{2}(t-u)^{2}-2 s(t+u)^{3}-(t+u)^{2}\left(3 t^{2}+2 t u+3 u^{2}\right)\right] \\
& \left.-(s+t+u)(t+u)^{2}\left(t^{2}+u^{2}\right)\right\}, \\
& \frac{d \sigma}{d t}\left(q \bar{q} \rightarrow Q \bar{Q}\left[{ }^{3} P_{J}^{(8)}\right] Z\right)=\frac{16 \pi \alpha_{s}^{2} g^{2} v_{Q}^{2}}{9 M^{3} s^{3}\left(2 m_{Z}^{2}-t-u\right)^{4}}\left\{16 m_{Z}^{8}-8 m_{Z}^{6}[8 s+5(t+u)]+2 m_{Z}^{4}\left[16 s^{2}+48 s(t+u)+23 t^{2}+38 t u+23 u^{2}\right]\right. \\
& +2 m_{Z}^{2}\left[8 s^{3}-4 s^{2}(t+u)-23 s(t+u)^{2}-(t+u)\left(13 t^{2}+14 t u+13 u^{2}\right)\right]+(t+u)\left[8 s^{2}(t+u)\right. \\
& \left.\left.+4 s\left(3 t^{2}+4 t u+3 u^{2}\right)+7(t+u)\left(t^{2}+u^{2}\right)\right]\right\}, \\
& \frac{d \sigma}{d t}\left(d \bar{u} \rightarrow Q \bar{Q}\left[{ }^{3} S_{1}^{(8)}\right] W^{-}\right)=\frac{d \sigma}{d t}\left(u \bar{d} \rightarrow Q \bar{Q}\left[{ }^{3} S_{1}^{(8)}\right] W^{+}\right)=\frac{\pi \alpha_{s}^{2} g^{\prime}{ }^{2}\left|V_{u d}\right|^{2}}{27 M^{3} s^{2} t^{2} u^{2}}\left\{m_{W}^{4}\left(t^{2}+u^{2}\right)-m_{W}^{2}(s+t+u)\left(t^{2}+u^{2}\right)\right. \\
& \left.+t u\left[2 s^{2}+2 s(t+u)+t^{2}+u^{2}\right]\right\} .
\end{aligned}
$$

[1] W.E. Caswell and G.P. Lepage, Phys. Lett. 167B, 437 (1986); G.T. Bodwin, E. Braaten, and G.P. Lepage, Phys. Rev. D 51, 1125 (1995); 55, 5853(E) (1997).

[2] E.L. Berger and D. Jones, Phys. Rev. D 23, 1521 (1981); R. Baier and R. Rückl, Phys. Lett. 102B, 364 (1981); Z. Phys. C 19, 251 (1983); B. Humpert, Phys. Lett. B 184, 105 (1987); R. Gastmans, W. Troost, and T.T. Wu, ibid. 184, 257 (1987); Nucl. Phys. B291, 731 (1987).

[3] E. Braaten and S. Fleming, Phys. Rev. Lett. 74, 3327 (1995); E. Braaten and T.C. Yuan, Phys. Rev. D 52, 6627 (1995); P. Cho and A.K. Leibovich, ibid. 53, 150 (1996); 53, 6203 (1996).

[4] CDF Collaboration, F. Abe et al., Phys. Rev. Lett. 69, 3704 (1992); 71, 2537 (1993); 79, 572 (1997); 79, 578 (1997); D0 Collaboration, S. Abachi et al., Phys. Lett. B 370, 239 (1996); D0 Collaboration, B. Abbott et al., Phys. Rev. Lett. 82, 35 (1999).

[5] E. Braaten, S. Fleming, and T.C. Yuan, Annu. Rev. Nucl. Part. Sci. 46, 197 (1996); B.A. Kniehl and G. Kramer, Phys. Lett. B 413, 416 (1997); M. Krämer, Prog. Part. Nucl. Phys. 47, 141 (2001).

[6] M. Beneke and M. Krämer, Phys. Rev. D 55, 5269 (1997); A.K. Leibovich, ibid. 56, 4412 (1997); M. Beneke, M. Krämer, and M. Vänttinen, ibid. 57, 4258 (1998); B.A. Kniehl and J. Lee, ibid. 62, 114027 (2000); S. Fleming, I.Z. Rothstein, and A.K. Leibovich, ibid. 64, 036002 (2001).

[7] E. Braaten, B.A. Kniehl, and J. Lee, Phys. Rev. D 62, 094005 (2000).
[8] Š. Todorova-Nová, in Proceedings of the XXXI International Symposium on Multiparticle Dynamics (XXXI-ISMD), Datong, China, 2001, edited by B. Yuting, Y. Meiling, and W. Yuanfang, eConf C010901, hep-ph/0112050; M. Chapkin, in Proceedings of the 7th International Workshop on Meson Production, Properties and Interaction (Meson 2002), Cracow, Poland, 2002 (World Scientific, Singapore, in press).

[9] M. Klasen, B.A. Kniehl, L.N. Mihaila, and M. Steinhauser, Phys. Rev. Lett. 89, 032001 (2002).

[10] J.P. Ma, B.H.J. McKellar, and C.B. Paranavitane, Phys. Rev. D 57, 606 (1998).

[11] G. Japaridze and A. Tkabladze, Phys. Lett. B 433, 139 (1998).

[12] M. Klasen, B.A. Kniehl, L. Mihaila, and M. Steinhauser, Nucl. Phys. B609, 518 (2001).

[13] R.M. Godbole, D. Indumathi, and M. Krämer, Phys. Rev. D 65, 074003 (2002).

[14] T. Mehen, Phys. Rev. D 55, 4338 (1997).

[15] M. Cacciari, M. Greco, and M. Krämer, Phys. Rev. D 55, 7126 (1997).

[16] C.S. Kim, J. Lee, and H.S. Song, Phys. Rev. D 55, 5429 (1997).

[17] J. Andruszkow et al., "TESLA Technical Design Report, Part II: The Accelerator," edited by R. Brinkmann, K. Flöttmann, J. Rossbach, P. Schmüser, N. Walker, and H. Weise, Report No. DESY 2001-011, ECFA 2001-209, TESLA Report 2001-23, and TESLA-FEL 2001-05, p. II-1.

[18] B. Badelek et al., "TESLA Technical Design Report, Part VI: 
Appendices, Chapter 1: The Photon Collider at TESLA," edited by R. Klanner and V. Telnov [17], p. VI-1.

[19] H. Abramowicz et al., "TESLA Technical Design Report, Part VI: Appendices, Chapter 2: THERA: Electron-Proton Scattering at a cms energy of $\sim 1 \mathrm{TeV}$," edited by R. Klanner, U. Katz, M. Klein, and A. Levy [17], p. VI-99.

[20] E. Braaten, J. Lee, and S. Fleming, Phys. Rev. D 60, 091501 (1999).

[21] E.J. Williams, Proc R. Soc. London A139, 163 (1933); C.F. von Weizsäcker, Z. Phys. 88, 612 (1934).

[22] R.J. DeWitt, L.M. Jones, J.D. Sullivan, D.E. Willen, and H.W. Wyld, Jr., Phys. Rev. D 19, 2046 (1979); 20, 1751(E) (1979).

[23] P. Chen, Phys. Rev. D 46, 1186 (1992).

[24] P. Chen, T.L. Barklow, and M.E. Peskin, Phys. Rev. D 49, 3209 (1994).

[25] I.F. Ginzburg, G.L. Kotkin, V.G. Serbo, and V.I. Telnov, Nucl. Instrum. Methods Phys. Res. 205, 47 (1983).

[26] V.I. Telnov, Nucl. Instrum. Methods Phys. Res. A 294, 72 (1990).

[27] A. Petrelli, M. Cacciari, M. Greco, F. Maltoni, and M.L. Mangano, Nucl. Phys. B514, 245 (1998); F. Maltoni, M.L. Mangano, and A. Petrelli, ibid. B519, 361 (1998).

[28] Particle Data Group, K. Hagiwara et al., Phys. Rev. D 66, 010001 (2002).

[29] M. Glück, E. Reya, and I. Schienbein, Phys. Rev. D 60, 054019 (1999); 62, 019902(E) (2000).
[30] A.D. Martin, R.G. Roberts, W.J. Stirling, and R.S. Thorne, Eur. Phys. J. C 4, 463 (1998).

[31] CDF Collaboration, T. Affolder et al., Phys. Rev. Lett. 86, 3963 (2001).

[32] S. Frixione, M.L. Mangano, P. Nason, and G. Ridolfi, Phys. Lett. B 319, 339 (1993).

[33] J.A. Aguilar-Saavedra et al., "TESLA Technical Design Report, Part III: Physics at an $e^{+} e^{-}$Linear Collider," edited by R.-D. Heuer, D. Miller, F. Richard, and P. Zerwas [17], p. III-166.

[34] B.A. Kniehl and L. Zwirner, Nucl. Phys. B621, 337 (2002); B637, 311 (2002).

[35] B.A. Kniehl and G. Kramer, Eur. Phys. J. C 6, 493 (1999).

[36] E. Braaten and T.C. Yuan, Phys. Rev. D 50, 3176 (1994).

[37] In this sense, we disagree with the statement made in Ref. [15] that process (15) with $\mathrm{s}={ }^{1} S_{0},{ }^{3} P_{0},{ }^{3} P_{2}$ can produce collinear or infrared singularities. The triple-gluon vertex present in the contributing Feynman diagrams is innocuous because the virtual gluon has a timelike virtuality in excess of $m_{C}^{2}$.

[38] There are two obvious typographical errors in Eq. (8) of Ref. [14]: $\left(2 m_{c}^{2}\right)$ and the second appearance of $\left\langle O_{8}^{J / \psi}\left({ }^{3} S_{1}\right)\right\rangle$ should be replaced by $\left(2 m_{c}\right)^{2}$ and $\left\langle O_{8}^{J / \psi}\left({ }^{1} S_{0}\right)\right\rangle$, respectively.

[39] This observation does not support the assertion made in Ref. [15] that light-quark-initiated processes are strongly suppressed and can be safely neglected. 